\title{
Combination of unreliable evidence sources in intuitionistic fuzzy MCDM framework
}

\author{
Xiaodan Wang ${ }^{\text {a }}$, Jingwei Zhu ${ }^{\text {a }}$, Yafei Song ${ }^{\text {a,b,* }}$, Lei Lei ${ }^{\text {a }}$ \\ ${ }^{a}$ Air and Missile Defense College, Air Force Engineering University, Xi'an 710051, PR China \\ ${ }^{b}$ Troop No.95835 of PLA, PR China
}

\begin{abstract}
In this paper, we consider the combination of unreliable evidence sources in multiple criteria decision making (MCDM) framework in intuitionistic fuzzy environment. In the intuitionistic fuzzy MCDM framework, evidence sources can be combined based on intuitionistic fuzzy aggregation operators when reliability factors are known. Generalized intuitionistic fuzzy aggregation operators are applied to combine evidence sources with intuitionistic fuzzy reliability factors. Discounting operation on unreliable evidence bodies is also extended to deal with uncertain reliability factors. Then we define a combination operation on intuitionistic fuzzy values to combine evidence bodies in the intuitionistic fuzzy MCDM model. Finally the estimation of the reliability factors without prior knowledge is studied. Our proposed evaluation method is based on the principle of self-assessment. It is implemented by the probabilistic comparison between intuitionistic fuzzy values. Moreover, the proposed evaluation method is independent of the dissimilarity measure between basic probability assignments. Numerical examples demonstrate the performance of our combination rules and reliability estimation method.
\end{abstract}

Keywords: Dempster-Shafer theory of evidence; Atanassov's intuitionistic fuzzy set (AIFS); Multiple criteria decision making (MCDM); Reliability evaluation; Evidence combination

\section{Introduction}

Dempster-Shafer evidence theory, also called belief function theory, is an important tool for uncertainty reasoning and decision making [1]-[3]. It has been successfully applied to deal with the uncertain and imprecise data in many fields including classification [4][5], clustering [6]-[9] and information fusion [10][13][37], etc. In evidence theory, when all evidence sources are considered equally reliable, evidence combinations are implemented by the Dempster's rule which is famous for its commutativity, associativity, and low computation burden. Although Dempster's rule of combination is well-founded theoretically [14]-[16], its lack of robustness is considered as a roadblock by researchers in this field. This is because counter-intuitive results may be obtained in some cases, especially when there is high conflict among bodies of evidence (BOEs) [17][18]. Such results are harmful for decision-making.

Many works have been done to solve so-called counter-intuitive combination results. Researchers holding two major viewpoints have debated for decades. The focus of the disputation lies on the cause of counter-intuitive results. The first viewpoint is that the counter-intuitive results are caused by Dempster's rule of combination, especially by its normalization step. Thus, a number of researchers have proposed alternative combination rules [19]-[23] based on various strategies to redistribute the conflict and provided fusion tools with the hope of obtaining more satisfactory results.

The second viewpoint is that the counter-intuitive results are caused by unreliable BOEs to be combined. According to this viewpoint, mass functions should be modified before combination occurs [18][24]-[26]. Evidence discounting and weighted averaging are two main methods for evidence modification. Many works on the discounting methods and weighted averaging for the unreliable sources of evidence have emerged to

\footnotetext{
* Corresponding author. Tel.: +86-13488130880.

E-mail addresses: yafei_song@163.com (Y.Song), afeu_wang@163.com (X. Wang).
} 
address this problem. The weighted averaging method essentially depends on the convex combination of all evidence bodies by assigning a weight factor to each evidence body, and all weights sum to one. The basic idea of the discounting method is that if one source of evidence is not fully reliable, it should be discounted by multiplying each basic probability mass by a factor, and assigning the left mass to the full set. In both methods, the reliability of evidence source is applied to evaluate the weight factor or the discounting factor.

Although both two types of viewpoints are rational, we prefer the idea that unreliable sources are the cause for the counter-intuitive results. In our view, the problem of Dempster's combination rule comes from its assumption that all sources of evidence are fully reliable. In fact, sources of information in real systems are always unreliable because different sources have access to different sectors of knowledge and experience. For example, experts differ in their level of expertise; some of them are more reliable than others due to their better knowledge, training, experience, intelligence, etc. To express their opinions, experts may use different background, methodology and knowledge. Hence, it is necessary to consider the experts' reliability and their judgments must be appropriately modified. Analogously, information provided by sensors does not have the same degree of reliability. This may be due not only to the same reasons as already mentioned for experts, but also to other factors more specific to sensors. For instance, measurements can differ from one sensor to another in terms of completeness, precision, and certainty. Additionally, the working environment can also affect the sensor reliability, since some of them could be better adapted to the conditions encountered in the considered environment than others.

Therefore, evidence bodies to be combined should be modified according to the reliability of their sources, which reflects the ability of each source to provide a correct assessment of the given problem. The effects of evidence from more reliable sources should be strengthened, while the effects of evidence from less reliable sources should be weakened. Thus, the evidence reliabilities must be assessed before combination.

This leaves us with the question of how to determine the reliability of evidence sources. It is noteworthy that terminologies of evidence reliability, sensor reliability, and reliability of evidence source are referred to the same meaning in the information fusion system based on evidence theory. Thus, these terms are used interchangeably throughout this paper.

When the prior information is available, the reliability of evidence source can be evaluated by training or optimization. Elouedi et al. [33] have proposed a method of assessing the sensor reliability in a classification problem based on the transferable belief model (TBM), an extension of evidence theory. In this method, the sensor reliability is assessed by minimizing the mean square error between the discounted sensor readings and the actual values of data.

The crucial problem in this approach is how to access the reliability of each evidence source when there is no prior information. As mentioned above, the reliability if evidence is related to the ability of each source to provide correct or creditable information. Without prior information, the training and optimization methods cannot make sense. Such situations have been regularly studied, and many methods have been proposed based on the principle of majority. By such principle, the dissent or disagreement among different pieces of evidence can be used to generate a measure of reliability as a weight factor or discounting factor. If one source of evidence has great (small) dissimilarity to the other sources, its reliability should be low (high). The evidential distance measure and evidential conflict measure are usually used to describe the difference or disagreement among different pieces of evidence. Therefore some approaches have been proposed to modify BOEs based on distance of evidence and evidential conflict [26][27].

Guo et al. [34] extended Elouedi's work in two aspects. On one hand, they developed a new evaluation method to improve Elouedi's method and called it the static evaluation method. On the other hand, they 
treated the evaluation task as a two-stage training process, namely, supervised (or static) and unsupervised (or dynamic) evaluation, respectively, and then proposed to combine them. This leads to a deeper insight into the issue of sensor reliability evaluation. The first one is what they have called the static supervised evaluation method. A static discounting factor assigned to a sensor is based on the comparison between its original readings and the actual values of data. Information content contained in the actual values of each target is extracted to determine its influence on the evaluation. This method also permits the evaluation of the reliability of the fusion result. The second one is the dynamic evaluation method, which can be used to dynamically evaluate the evidence reliability by adaptive learning and regulation in real-time situations. The dynamic reliability is related to the contexts of sensor acquisitions and sensor dynamic performance.

In fact, the dynamic reliability evaluation mentioned above is implemented in the situation of lacking a priori knowledge, which is more common in practice. To solve this problem, the "principle of majority" is always taken as an appropriate choice. As interpreted by Guo et al. in [34], the evidential distance measure, conflict measure, and other induced dissimilarity measures are used to evaluate evidence reliability. By this principle, many dynamic reliability evaluation methods have been proposed. For example, Schubert [38] proposed a degree of falsity based on which the BOEs could be discounted. Based on Jousselme's [36] distance measure, Klein and Colot [35] propose the degree of dissent, which is evaluated by comparing a basic probability assignment (BBA) to the average BBA in a set. The distance between a BPA and the average BPA is applied to estimate its reliability. Based on Jousselme's distance measure [36]and Schubert's idea [38], Yang et al. [27] defined a new disagreement measure by borrowing ideas from the design of Schubert's degree of falsity to estimate the reliability of evidence source. Liu et al. [37] noted that the distance represented the difference between BPAs, whereas the conflict coefficient revealed the divergence degree of the hypotheses that two belief functions strongly support. These two aspects of dissimilarity were complementary in a certain sense, and their fusion could be used as the dissimilarity measure. So they presented a new dissimilarity measure by fusing distance and conflict measure based on Hamacher T-conorm fusion rule. In the evaluation of reliability of a source, both its dissimilarity with other sources and their reliability factors were considered.

However, taking a closer examine on these methods, we can find that they all boil down to the definition of dissimilarity measure. In other words, the definition of dissimilarity measure largely determines the evaluation of evidence reliability. When reliability of each evidence source has been evaluated, original evidence bodies are modified by discounting operation or weighted averaging operation. Then, the modified BPAs are combined by Dempster's combination rule. Although these methods can reduce the influence from unreliable evidence sources, these combination results may not suitable for decision making due to the probability assigned other non-singleton subsets. So transformation from BPA to probability distribution is needed in decision-making step. Nevertheless, the probabilistic transformation of BPAs is still an open topic. Moreover, most existing modifying operations on evidence bodies cannot deal with uncertain reliability factors (interval value or intuitionistic fuzzy value). Thus, generalized modifying operations are necessary.

To alleviate aforementioned problems and propose a new viewpoint for evidence combination, we will consider the combination of unreliable evidence sources in the intuitionistic fuzzy multiple criteria decision making (MCDM) framework. Multiple criteria decision making refers to making decision for alternatives in the presence of multiple and conflicting criteria [39], it has attracted much attention from researchers in the community of decision making [40]-[43]. In this paper, we will transform the problem of evidence combination to the mode of MCDM under intuitionistic fuzzy environment. In the MCDM framework, novel combination rules, discounting operations and reliability evaluation methods will be proposed based on intuitionistic fuzzy operations. 
Our perspective is that the combination of evidence from different sources is similar to multiple criteria decision making. Under such assumption, we can build MCDM model based on evidence sources to be combined. The main assumption of this study relies on the relation between evidence theory and Atanassov's intuitionistic fuzzy sets (AIFSs). By transforming BPAs to AIFSs, evidence combination can be regarded as a multiple criteria decision making (MCDM) problem under intuitionistic fuzzy environment. In the problem of classification based on multi-sensor data fusion using evidence theory, the readings of each sensor represents certain part of characteristics of the target, i.e., different sensors represent different criteria. Let $\Omega=\left\{A_{1}, A_{2}, \cdots, A_{m}\right\}$ be the discernment frame. The Bayesian BPA $m_{i}\left(A_{j}\right)$ coming from sensor $i$ can be interpreted as the value of alternative $A_{j}$ with respect to the criterion $i$. This discussion of sensor fusion is also potentially applicable to other kind of evidence combination problems. So we can estimate the reliability of evidence source borrowing idea of evaluating weights of criteria in MCDM model. Based on the reliability factor of each evidence source, the BPA obtained from them can be discounted or weighting averaged. Moreover, in the frame of intuitionistic fuzzy MCDM, it is convenient to combine unreliable evidence sources based on intuitionistic fuzzy aggregation operators when reliability factors are known. Additionally, since evidence reliability factors may be uncertain, generalized discounting operation on evidence bodies is desirable to address this problem.

We note that Frikha [63] has made use of a multiple criteria approach for reliability estimation in belief function theory. However, his focus is different from ours. He claimed that the assessment of expert information based on a single criterion is not reliable enough since the mono-criterion approach is often insufficient to reflect reality. He took both conflict between expert judgments and the imperfection of expert information into consideration during the calculation of reliability degrees. Then he opted for the use of the Multiple Criteria Decision Aid (MCDA) to estimate the reliability degrees of expert judgments. Our focus is on the representation of information about an uncertain variable and on estimating the reliability degrees based on the MCDM model in intuitionistic environment. The combination of unreliable evidence sources is also considered. We do not take the position that approach suggested in the paper is the best or the only suitable approach to evaluate reliability of evidence sources, or to combine evidence sources; our position is that this is a reasonable approach that may be of use to some decision makers.

To facilitate our discussion, in Section 2, we first describe background knowledge related to Dempster-Shafer theory of evidence, Atanassov's intuitionistic fuzzy sets and multiple criteria decision making methods. In Section 3, we review and critically analyze existing approaches of reliability evaluation for evidence sources. Combination of unreliable evidence sources based on MCDM is developed in Section 4. We propose a dynamic reliability evaluation method based on self-assessment in Section 5. Numerical examples are given to illustrate the performance of the proposed approaches in Section 6. We come to the conclusion of this paper in Section 7.

\section{Preliminaries}

The background knowledge presented in this section deals with the following three main points: (1) the interpretation of Dempster-Shafer theory of evidence, (2) preliminary knowledge on AIFSs, and (3) the multiple criteria decision making model in intuitionistic fuzzy environment, which will facilitate the reliability estimation of evidence sources.

\subsection{Dempster-Shafer theory of evidence}

Dempster-Shafer theory of evidence is an approach for uncertainty reasoning. It enables us to combine evidence from different sources and arrive at a degree of belief. It has become an important method for the study of information fusion. To facilitate our discussion, we will first briefly review basic concepts of the 
evidence theory, the combination rule and its counter-intuitive aspect. We then introduce the discounting strategy for unreliable evidence.

\subsubsection{Basic concepts}

Dempster-Shafer theory of evidence is modeled based on a finite set of mutually exclusive elements, called the frame of discernment denoted by $\Omega$. The power set of $\Omega$, denoted by $2^{\Omega}$, contains all subsets of $\Omega$, including $\Omega$ itself and empty set $\varnothing$. Singleton sets in a frame of discernment $\Omega$ will be called atomic sets because they do not contain nonempty subsets. It is assumed that only one atomic set can be true at any one time. If a set is assumed to be true, then all supersets are considered true as well.

An observer who believes that one or several sets in the power set of $\Omega$ might be true can assign belief masses to these sets. Belief mass on an atomic set $A \in 2^{\Omega}$ is interpreted as the belief that the set in question is true. Belief mass on a non-atomic set $A \in 2^{\Omega}$ is interpreted as the belief that one of the atomic sets it contains is true, but that the observer is uncertain about which of them is true. The following definitions are significant in Dempster-Shafer theory.

Definition 2.1 [1]. Let $\Omega=\left\{A_{1}, \cdots, A_{n}\right\}$ be the frame of discernment. A basic probability assignment (BPA) is a function $m: 2^{\Omega} \rightarrow[0,1]$, satisfying the two following conditions:

$$
\begin{gathered}
m(\varnothing)=0 \\
\sum_{A \subseteq \Omega} m(A)=1
\end{gathered}
$$

Such a function is also called belief structure. For each subset $A \subseteq \Omega$, the value taken by the BPA at $A$ is called the basic probability mass of $A$, denoted by $m(A)$. The value $m(A)$ can be interpreted as the mass or the proportion of all relevant and available evidence that supports the claim that a particular element of $\Omega$ belongs to the set $A$ but to no particular subset of $A$. The mass of the empty set is 0 , and the summation of the masses of all subsets of $\Omega$ is 1 . The value of 0 indicates total non-belief in a hypothesis, while the value of 1 indicates total belief. The mass of $\Omega$, denoted as $m(\Omega)$, indicates the amount of uncertainty, called ignorance.

Definition 2.2 [1]. A subset $A$ of $\Omega$ is called a focal element of a belief structure if $m(A)>0$.

Definition 2.3 [2]. A Bayesian belief structure (BBS) on $\Omega$ is a belief structure on $\Omega$ whose focal elements are atomic sets (singletons) of $\Omega$.

A Bayesian belief structure is also called as a Bayesian BPA. It is identical to the probability distribution on $\Omega$.

Associated with the measure $m$ is a dual measure that plays a significant role in the fusion of information. One part of the dual measure is the belief function, denoted as Bel, which is a mapping from $2^{\Omega}$ to $[0,1]$ measuring the total mass that must be distributed, in some way, among the elements of a certain subset of $\Omega$. Thus, we have the definition of belief function as:

Definition 2.4 [2]. Given a belief structure $m$ on $\Omega$, the belief function which is in one-to-one correspondence with $m$ can be defined as:

$$
\operatorname{Bel}(A)=\sum_{B \subseteq A} m(B)
$$

In particular, the belief associated with $A$ can be interpreted as the sum of the proportions of the total belief (masses) in all elements which entail $A$.

Another important function is the plausibility function, denoted as $P l$, which is also a mapping from $2^{\Omega}$ to $[0,1]$. The plausibility function measures the maximal amount of mass that can be distributed among the elements in certain subset of $\Omega$. Thus, the definition of plausibility function is expressed as:

Definition 2.5 [2]. Given a belief structure $m$ on $\Omega$, the plausibility function of the element $A$ is: 


$$
P l(A)=\sum_{B \cap A \neq \varnothing} m(B)
$$

$\operatorname{Bel}(A)$ and $P l(A)$ are the lower limit and upper limits of the belief level of hypothesis $A$, respectively. The belief value of hypothesis $A$ is regarded as the minimal uncertainty about $A$, and its plausibility value is regarded as the maximal uncertainty about $A$. The relation between them is:

$$
\begin{gathered}
\operatorname{Pl}(A)=1-\operatorname{Bel}(\bar{A}) \\
P l(A) \geq \operatorname{Bel}(A)
\end{gathered}
$$

Where $\bar{A}$ is the classical complement of hypothesis $A$.

$[\operatorname{Bel}(A), P l(A)]$ is the confidence interval which describes the uncertainty about $A$, and $\operatorname{Pl}(A)-\operatorname{Bel}(A) \quad$ represents the level of ignorance about $A$. If the difference between $\operatorname{Bel}(A)$ and $\operatorname{Pl}(A)$ increases, then the information for fusion decreases or becomes unreliable. The difference provides a measurement of uncertainty about the level of belief in a decision. The interval $[0, \operatorname{Bel}(A)]$ is the range of support for hypothesis $A$, while the interval $[0, P l(A)]$ is the range of quasi belief in $A$, whereas the interval $[P l(A), 1]$ is the range of refusal of $A$.

An uncertainty range delimits the upper and lower bounds for the degree of belief in hypothesis $A$. Some special belief ranges have been defined as follows:

(1) $[1,1]$ represents that hypothesis $A$ is true;

(2) $[0,0]$ represents that hypothesis $A$ is false;

(3) $[0,1]$ represents that hypothesis $A$ is totally unknown.

In evidence theory, we need to transform BPA to probability distribution on the discernment frame for the convenience of decision making. Pignistic transformation proposed by Smets [21] is widely used. The pignistic transformation maps a BPA $m$ to so called pignistic probability function. It is defined as following.

Definition 2.6 [21]. The pignistic transformation of BPA $m$ on $\Omega=\left\{A_{1}, \ldots, A_{n}\right\}$ is given by

$$
\operatorname{BetP}(A)=\sum_{B \subseteq \Omega} \frac{|A \cap B|}{|B|} \frac{m(B)}{1-m(\varnothing)}, \forall A \subseteq \Omega
$$

where $|\cdot|$ is the cardinality of a set.

\subsubsection{Dempster's rule of combination}

Definition 2.7 [1]. Given two belief structures $m_{1}$ and $m_{2}$ on $\Omega$, the belief structure that results from the application of Dempster's combination rule, denoted as $m_{1} \oplus m_{2}$, or $m_{12}$ for short, is given by:

$$
m_{12}(A)=\left\{\begin{array}{l}
\frac{\sum_{B \cap C=A} m_{1}(B) m_{2}(C)}{1-\sum_{B \cap C=\varnothing} m_{1}(B) m_{2}(C)}, \forall A \subseteq \Omega, A \neq \varnothing \\
0, A=\varnothing
\end{array}\right.
$$

When multiple independent sources of evidence are available, the combined evidence can be obtained as:

$$
m(A)=\left\{\begin{array}{l}
\frac{\sum_{\cap A_{i}=A} \prod_{i=1}^{n} m_{i}\left(A_{i}\right)}{1-\sum_{n A_{i}=\varnothing} \prod_{i=1}^{n} m_{i}\left(A_{i}\right)}, \forall A \subseteq \Omega, A \neq \varnothing \\
0, A=\varnothing
\end{array}\right.
$$

Here, $n$ is the number of evidence pieces in the process of combination, $i$ denotes the $i$ th piece of evidence, $m_{i}\left(A_{i}\right)$ is the BPA of hypothesis $A_{i}$ supported by evidence $i$. The value $m(A)$ reflects the degree of combined support, joint mass, from $n$ mutually independent sources of evidence corresponding 
to $m_{1}, m_{2}, \cdots, m_{n}$, respectively. The quantity $k$ defined in Eq. (10) is the amount of conflict among $n$ mutually independent pieces of evidence, which is equal to the mass of the empty set after the conjunctive combination and before the normalization step. It represents contradictory evidence.

$$
k=\sum_{n A_{i}=\varnothing} \prod_{i=1}^{n} m_{i}\left(A_{i}\right)
$$

The value $k=0$ corresponds to the absence of conflicts among the evidence from different sources, whereas $k=1$ implies complete contradiction among the evidences. Indeed $k=0$, if and only if no empty set is created when all evidences are combined, and $k=1$ if and only if all the sets resulting from this combination rule are empty sets. The weight of partial conflict is $\prod_{i=1}^{n} m_{i}\left(A_{i}\right)$ with $\cap A_{i}=\varnothing$. The global conflict $k$ is then the sum of all partial conflicts.

Dempster's rule, however, has an inherent problem. When the pieces of evidence are completely contradictory, i.e. $k=1$, combination cannot be performed. When they are highly conflicting, i.e. $k \rightarrow 1$, the combination results seldom agree with the actual situation, and are counter-intuitive (see the example given by Zadeh [17]).

Example 2.1. Consider a situation in which we have two belief structures $m_{1}$ and $m_{2}$ over the same frame of discernment $\Omega=\{A, B, C\}$. Let these two structures be as follows:

$$
\begin{aligned}
& m_{1}: m_{1}(A)=0.9, m_{1}(B)=0.1, \\
& m_{2}: m_{2}(B)=0.1, m_{2}(C)=0.9 .
\end{aligned}
$$

Applying Dempster's rule to these structures yields $m(A)=m(C)=0, m(B)=1$. We can see that $m_{1}$ and $m_{2}$ have low support level to hypothesis $B$, but the resulting BPA has complete support to $B$. On the other hand, $m_{1}$ and $m_{2}$ have high support level on hypotheses $A$ and $C$, respectively, but $A$ and $C$ are totally unbelievable in the result. This appears to be counter-intuitive.

The reason for such counter-intuitive behavior is that Dempster's rule cannot handle highly conflicting evidence. Such problem can be handled from two main points of view. If the counter-intuitive behavior is believed to be caused by unreliable evidences, then the evidences should be discounted. If the counter-intuitive behavior is attributed to the combination rule, improvements of the combination rule, as done in several studies [20]-[27], should then be made.

\subsubsection{Discount on evidence}

When a source of evidence is only partially reliable to a known reliability degree $\lambda \in[0,1]$, a discounting operation can be defined on the associated BPA [22]. The most common discounting operation was first introduced by Shafer in [2]. The discounting operation is given by

$$
\left\{\begin{array}{l}
m^{\prime}(A)=\lambda m(A), A \subset \Omega \\
m^{\prime}(\Omega)=1-\lambda+\lambda m(\Omega)
\end{array}\right.
$$

where $\lambda$ represents the degree of reliability of the evidence. If $\lambda=1$, i.e., the evidence is completely reliable, the BPA will remain unchanged. If $\lambda=0$, i.e., the evidence is completely unreliable, then the BPA will become $m(\Omega)=1$, which means that the evidence is fully ignorance for decision-making.

\subsection{Atanassov's intuitionistic fuzzy sets}

\subsubsection{Definitions related to AIFSs}

Since Atanassov's intuitionistic fuzzy set can be considered as a generation of Zadeh's fuzzy set, we first give the definition of Zadeh's fuzzy set, followed by brief description on basic concepts of AIFSs.

Definition 2.8 [28]. Let $X=\left\{x_{1}, x_{2}, \cdots, x_{n}\right\}$ be a universe of discourse, then a fuzzy set $A$ in $X$ is 
defined as follows:

$$
A=\left\{\left\langle x, \mu_{A}(x)\right\rangle \mid x \in X\right\}
$$

where $\mu_{A}(x): X \rightarrow[0,1]$ is the membership degree.

Definition 2.9 [29][30]. An Atanassov's intuitionistic fuzzy set $A$ in $X$ can be written as:

$$
A=\left\{\left\langle x, \mu_{A}(x), v_{A}(x)\right\rangle \mid x \in X\right\}
$$

where $\mu_{A}(x): X \rightarrow[0,1]$ and $v_{A}(x): X \rightarrow[0,1]$ are membership degree and non-membership degree, respectively, with the condition:

$$
0 \leq \mu_{A}(x)+v_{A}(x) \leq 1
$$

$\pi_{A}(x)$ determined by the following expression:

$$
\pi_{A}(x)=1-\mu_{A}(x)-v_{A}(x)
$$

is called the hesitancy degree of the element $x \in X$ to the set $A$, and $\pi_{A}(x) \in[0,1], \forall x \in X$.

$\pi_{A}(x)$ is also called the intuitionistic index of $x$ to $A$. Greater $\pi_{A}(x)$ indicates more vagueness on $x$. Obviously, when $\pi_{A}(x)=0, \forall x \in X$, an AIFS degenerates to Zadeh's fuzzy set.

It is worth noting that besides Definition 2.9 there are other possible representations of AIFSs proposed in the literature [31][32]. Atanassov and Gargov [32] proposed to use an interval representation $\left[\mu_{A}(x), 1-v_{A}(x)\right]$ of AIFS $A$ in $X$ instead of pair $\left\langle\mu_{A}(x), v_{A}(x)\right\rangle$. This approach is equivalent to the interval valued fuzzy sets interpretation of AIFS, where $\mu_{A}(x)$ and $1-v_{A}(x)$ represent the lower bound and upper bound of membership degree, respectively. Obviously, $\left[\mu_{A}(x), 1-v_{A}(x)\right]$ is a valid interval, since $\mu_{A}(x) \leq 1-v_{A}(x)$ always holds for $\mu_{A}(x)+v_{A}(x) \leq 1$.

In the sequel, $\operatorname{AIFSs}(X)$ denotes the set of all AIFSs in $X$. If $|X|=1$, i.e., there is only one element $x$ in $X$, the AIFS $A$ in $X$ usually is denoted by $A=\left\langle\mu_{A}, v_{A}\right\rangle$ for short, which is also called an intuitionistic fuzzy value (IFV). Moreover, it is clear that each IFV $A=\left\langle\mu_{A}, v_{A}\right\rangle$ corresponds to an interval-valued value $\left[\mu_{A}, 1-v_{A}\right]$. If $\mu_{A}=1-v_{A}$, the IFV reduces to a real number. The family of all IFVs will be denoted by $L^{*}[47]$.

In the AIFS theory, an AIFS $\left\langle\mu_{A}(x), v_{A}(x)\right\rangle$ has some physical interpretations. For example, if $\left\langle\mu_{A}(x), v_{A}(x)\right\rangle=\langle 0.2,0.3\rangle$, then the degree of indeterminacy $\pi_{A}(x)$ can be easily determined as 0.5 . These can be interpreted as "the degree that element $x$ belongs to $A$ is 0.2 , the degree that element $x$ does not belong to $A$ is 0.3 , and the degree that element $x$ belongs indeterminately to $A$ is 0.5 ". In a voting model, they can be interpreted as "the vote for resolution is two in favor and three against, with five abstentions" [48]. In addition, for a fuzzy set $B$ in $X$, since $v_{B}(x)=1-\mu_{B}(x)$, the indeterminacy degree of $x$ to $B$ can be expressed as $\pi_{B}(x)=1-\mu_{B}(x)-\left(1-\mu_{B}(x)\right)=0$. The fuzzy set is thus a particular case of the AIFS.

Definition 2.10 [30]. For $A \in \operatorname{AIFSs}(X)$ and $B \in \operatorname{AIFSs}(X)$, some relations between them are defined as:

(R1) $A>B$ iff $\forall x \in X \quad \mu_{A}(x)>\mu_{B}(x), v_{A}(x)<v_{B}(x)$;

(R2) $A=B$ iff $\forall x \in X \quad \mu_{A}(x)=\mu_{B}(x), v_{A}(x)=v_{B}(x)$; 
(R3) $A^{C}=\left\{\left\langle x, v_{A}(x), \mu_{A}(x)\right\rangle \mid x \in X\right\}$, where $A^{C}$ is the complement of $A$.

Definition 2.11 [45]. For two intuitionistic fuzzy values $A=\left\langle\mu_{A}, v_{A}\right\rangle$ and $B=\left\langle\mu_{B}, v_{B}\right\rangle$, the ordinary partial ordered relations are defined as: $A \geq B \Leftrightarrow \mu_{A} \geq \mu_{B}, v_{A} \leq v_{B} ; A=B \Leftrightarrow \mu_{A}=\mu_{B}, v_{A}=v_{B}$.

Following these relations, we can find that the smallest intuitionistic fuzzy value in $L^{*}$ is $\langle 0,1\rangle$ denoted by $\mathbf{0}$; while the largest element is $\langle 1,0\rangle$ denoted by $\mathbf{1}$.

Definition 2.12 [29]. Let $A=\left\{\left\langle x, \mu_{A}(x), v_{A}(x)\right\rangle \mid x \in X\right\}, B=\left\{\left\langle x, \mu_{B}(x), v_{B}(x)\right\rangle \mid x \in X\right\}$ be two AIFSs in the $X$, then the following operations can be defined:

$$
\begin{aligned}
& A \cap B=\left\{\left\langle x, \min \left(\mu_{A}(x), \mu_{B}(x)\right), \max \left(v_{A}(x), v_{B}(x)\right)\right\rangle \mid x \in X\right\}, \\
& A \cup B=\left\{\left\langle x, \max \left(\mu_{A}(x), \mu_{B}(x)\right), \min \left(v_{A}(x), v_{B}(x)\right)\right\rangle \mid x \in X\right\}, \\
& A \oplus B=\left\{\left\langle x, \mu_{A}(x)+\mu_{B}(x)-\mu_{A}(x) \cdot \mu_{B}(x), v_{A}(x) \cdot v_{B}(x)\right\rangle \mid x \in X\right\}, \\
& A \otimes B=\left\{\left\langle x, \mu_{A}(x) \cdot \mu_{B}(x), v_{A}(x)+v_{B}(x)-v_{A}(x) \cdot v_{B}(x)\right\rangle \mid x \in X\right\}, \\
& \gamma \cdot A=\left\{\left\langle x, 1-\left(1-\mu_{A}(x)\right)^{\gamma},\left(v_{A}(x)\right)^{\gamma}\right\rangle \mid x \in X\right\}, \\
& A^{\gamma}=\left\{\left\langle x,\left(\mu_{A}(x)\right)^{\gamma}, 1-\left(1-v_{A}(x)\right)^{\gamma}\right\rangle \mid x \in X\right\} .
\end{aligned}
$$

\subsubsection{Ranking method of IFVs}

In real-life decision situations, comparing or ranking IFVs is an important problem. Since Definition 2.11 can be used to rank IFVs $A$ and $B$ satisfying $\mu_{A} \geq \mu_{B}, v_{A} \leq v_{B}$, other IFVs cannot be ranked by this rule. Here we introduce and employ the ranking method based on the score function and the accuracy function, which are defined as follows.

Definition 2.13 [49][50]. Let $A=\left\langle\mu_{A}, v_{A}\right\rangle$ be an IFV. Then the score function $s$ and the accuracy function of $a$ can be mathematically expressed as: $s(A)=\mu_{A}-v_{A}$ and $a(A)=\mu_{A}+v_{A}$, respectively.

It is easily derived from Definition 2.13 that $s(A) \in[-1,1]$ and $a(A) \in[0,1]$ for any AIFS $A$.

$s(A)$ reflects the difference of the certainty degree to which one element belongs to the AIFS $A$. The larger $s(A)$ the greater $A$.

$a(A)$ expresses the sum of the certainty degree to which one element does not belong to the AIFS $A$. Larger $a(A)$ indicated that $A$ is more accurate.

It is easy to see that the meanings of the score function and the accuracy function are similar to those of the mean and variance in Statistics, respectively. Thus, using the score function and the accuracy function, a ranking method between two IFVs can be established as follows.

Definition 2.14 [50]. Let $A=\left\langle\mu_{A}, v_{A}\right\rangle$ and $B=\left\langle\mu_{B}, v_{B}\right\rangle$ be two IFVs. Designate:

(1) If $s(A)>s(B), A$ is bigger than $B$, denoted by $A \succ B$;

(2) When $s(A)=s(B)$, we have: 
(i) If $a(A)=a(B), \quad A$ is equal to $B$, denoted by $A=B$;

(ii) If $a(A)<a(B), A$ is smaller than $B$, denoted by $A \prec B$;

(iii) If $a(A)>a(B), A$ is bigger than $B$, denoted by $A \succ B$.

Example 2.2. Assume that there are three IFVs $A_{1}=\langle 0.6,0.3\rangle, A_{2}=\langle 0.45,0.2\rangle$, and $A_{3}=\langle 0.5,0.25\rangle$.

According to Definition 2.13, scores of the IFVs $A_{1}, A_{2}$ and $A_{3}$ are computed as follows:

$s\left(A_{1}\right)=0.6-0.3=0.3, \quad s\left(A_{2}\right)=0.45-0.2=0.25, \quad s\left(A_{3}\right)=0.5-0.25=0.25$.

Hence, $A_{1} \succ A_{2}=A_{3}$ if the scores of the IFVs $A_{1}, A_{2}$ and $A_{3}$ are merely taken into consideration. In order to make a distinction between $A_{2}$ and $A_{3}$, according to Definition 2.13, accuracy values of the IFVs $A_{2}$ and $A_{3}$ are obtained as follows:

$a\left(A_{2}\right)=0.45+0.2=0.65, a\left(A_{3}\right)=0.5+0.25=0.75$.

Then, according to Definition 2.14, it is easy to see that $A_{1} \succ A_{3} \succ A_{2}$.

\subsection{Intuitionistic fuzzy MCDM model}

Suppose that there exists an alternative set $A=\left\{A_{1}, A_{2}, \cdots, A_{m}\right\}$ which consists of $m$ efficient alternatives from which the best alternative has to be selected. Alternatives are assessed on $n$ criteria. Denote the set of all criteria by $X=\left\{x_{1}, x_{2}, \cdots, x_{n}\right\}$. Usually alternatives are evaluated on quantitative criteria and qualitative criteria, respectively. Generally, alternatives are evaluated on quantitative criteria by using numerical values while they are evaluated on qualitative criteria through using IFVs. In the intuitionistic fuzzy environment, numerical values need to be transformed to IFVs for consistency and clarity. So we need not only to acquire IFVs of alternatives on qualitative criteria but also transform numerical values of alternatives on quantitative criteria into IFVs. This is beyond the scope of this paper; hence, it is not presented here. In light of this, we point the reader to some references that may provide the basis for alternative approaches to address this issue [50][51].

By expressing values of alternatives on qualitative and quantitative criteria as AIFSs in a unified way, the vector of IFVs of all $n$ criteria for alternatives $A_{i} \in A$ can be written, respectively, as:

$$
\left(r_{i 1}, r_{i 2}, \cdots, r_{i n}\right)=\left(\left\langle\mu_{i 1}, v_{i 1}\right\rangle,\left\langle\mu_{i 2}, v_{i 2}\right\rangle, \cdots,\left\langle\mu_{i n}, v_{i n}\right\rangle\right), \quad i=1,2, \cdots, m
$$

Thus a MCDM problem under intuitionistic fuzzy condition can be concisely expressed in the matrix format as follows:

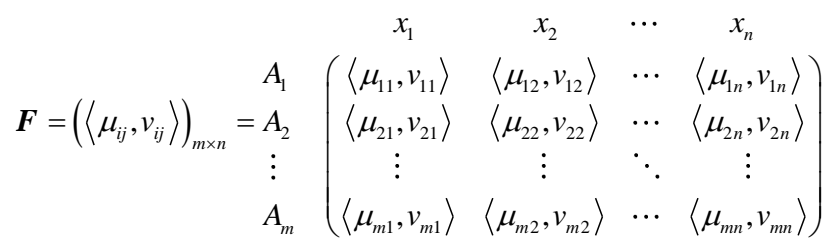

which is referred to as an intuitionistic fuzzy decision matrix used to represent the MCDM model in intuitionistic fuzzy environment.

\section{Combing unreliable evidence sources based on MCDM}

A multi-sensor data fusion system is an important component in many fields dealing with pattern recognition, identification, diagnosis, etc. It is used with the hope that the aggregation of several sensors achieves better results. Generally speaking, each sensor provides information of target identification from different aspects. If we suppose that each sensor represents a feature or criterion of the target, the readings of each sensor can be regarded as the degree to which the target matched to each pattern in the discernment frame under such criterion. Apparently, it can be also taken as the evaluation of each alternative (pattern in 
the discernment frame) on the criterion. So the problem of information fusion is analogous to multiple criteria decision making.

As discussed earlier, in evidence theory, $[\operatorname{Bel}(A), P l(A)]$ is the confidence interval which describes the uncertainty about $A$. It can be used to define the lower and upper probability bounds of the imprecise probability of $A$. Here, $\operatorname{Bel}(A)$ is the lower probability, and $\operatorname{Pl}(A)$ is the upper probability. Thus, the probability $P(A)$ lies in an interval $[\operatorname{Bel}(A), P l(A)]$. We note $[\operatorname{Bel}(A), P l(A)]$ is a sub-interval the unit interval $[0,1]$. In the application of pattern, $\operatorname{Bel}(A)$ can be taken as the membership degree to which the object belongs to $A$, while $1-\operatorname{Pl}(A)$ is the non-membership degree. Based on such analysis, a belief structure $m$ on the discernment frame $\Omega=\left\{A_{1}, A_{2}, \cdots A_{p}\right\}$ can be transformed to an interval probability distribution on $A$. Considering the interval representation of IFV, the interval probabilities can be further switched to an intuitionistic fuzzy values $\left\langle\mu\left(A_{i}\right), v\left(A_{i}\right)\right\rangle$ with $\mu\left(A_{i}\right)=\operatorname{Bel}\left(A_{i}\right)$ and $v\left(A_{i}\right)=1-\operatorname{Pl}\left(A_{i}\right)$. For the sake of exposition, we call $\left\langle\mu\left(A_{i}\right), v\left(A_{i}\right)\right\rangle$ as the intuitionistic fuzzy probability of $A_{i}$. Therefore, the model of multiple criteria decision making model in the intuitionistic fuzzy environment can be established.

Let $\Omega=\left\{A_{1}, A_{2}, \cdots, A_{p}\right\}$ be the discernment frame. $m_{1}, m_{2}, \cdots, m_{q}$ are belief structures on $\Omega$. $\left\langle\mu_{j}\left(A_{i}\right), v_{j}\left(A_{i}\right)\right\rangle, i=1,2, \cdots, p, j=1,2, \cdots, q$ is the intuitionistic fuzzy probability of $A_{i}$ generated by $m_{j}$, where $\mu_{j}\left(A_{i}\right)=\operatorname{Bel}_{j}\left(A_{i}\right), \quad v_{j}\left(A_{i}\right)=1-P l_{j}\left(A_{i}\right)$. Then the decision matrix can be written as:

$$
\begin{aligned}
& \boldsymbol{F}=\left(\left\langle\mu_{j}\left(A_{i}\right), v_{j}\left(A_{i}\right)\right\rangle\right)_{p \times q} \\
& \begin{array}{llll}
m_{1} & m_{2} & \cdots & m_{q}
\end{array} \\
& \begin{array}{c}
A_{1} \\
A_{2} \\
\vdots \\
A_{p}
\end{array} \quad\left(\begin{array}{cccc}
A_{11} & A_{12} & \cdots & A_{1 q} \\
A_{21} & A_{22} & \cdots & A_{2 q} \\
\vdots & \vdots & \ddots & \vdots \\
A_{p 1} & A_{p 2} & \cdots & A_{p q}
\end{array}\right) \\
& \begin{array}{llll}
m_{1} & m_{2} & \cdots & m_{q}
\end{array}
\end{aligned}
$$

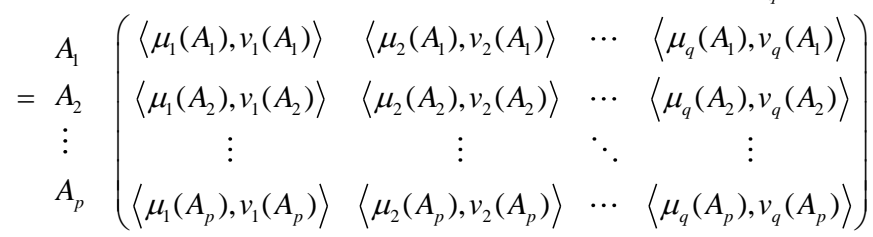

Our perspective on evidence combination is that combining evidence from different sources is similar to evaluating different alternatives in multiple criteria decision-making. When the reliability of each evidence source is known, based on the model of multiple criteria decision making in the intuitionistic fuzzy environment, the combination of unreliable evidence sources can be implemented by intuitionistic fuzzy aggregation operators. Thus, final decision can be achieved. Moreover, in the situation where the credibility factor needs to be determined, the reliability of evidence can be evaluated by assessing weights of criteria. In the following, we first propose combination methods for unreliable evidence sources, whose reliability factors are a priori given.

\subsection{Evidence combination based on intuitionistic fuzzy aggregation operators}

Based on intuitionistic fuzzy operations listed in Definition 2.12, a set of intuitionistic fuzzy aggregation operators have been proposed. Let $\alpha_{i}=\left\langle\mu_{\alpha_{i}}, v_{\alpha_{i}}\right\rangle \quad(i=1,2, \cdots, q)$ be a collection of intuitionistic fuzzy values, $\boldsymbol{w}=\left(w_{1}, w_{2} \cdots, w_{q}\right)^{T}$ be the aggregation-associated weight vector such that 
$w_{j} \in[0,1]$, and $\sum_{1}^{q} w_{j}=1$. Then we have the following aggregation operators.

(1) The intuitionistic fuzzy weighted averaging (IFWA) operator [44]:

$$
\begin{aligned}
& \operatorname{IFWA}\left(\alpha_{1}, \alpha_{2}, \cdots, \alpha_{q}\right) \\
& =\bigoplus_{i=1}^{q} w_{i} \alpha_{i}=w_{1} \alpha_{1} \oplus w_{2} \alpha_{2} \oplus \cdots \oplus w_{q} \alpha_{q} \\
& =\left\langle 1-\prod_{i=1}^{q}\left(1-\mu_{\alpha_{i}}\right)^{w_{i}}, \prod_{i=1}^{q} v_{\alpha_{i}}^{w_{i}}\right\rangle
\end{aligned}
$$

(2) The intuitionistic fuzzy weighted geometric (IFWG) operator [46]:

$$
\begin{aligned}
& \operatorname{IFWG}\left(\alpha_{1}, \alpha_{2}, \cdots, \alpha_{q}\right) \\
& =\bigotimes_{i=1}^{q}\left(\alpha_{i}^{w_{i}}\right)=\alpha_{1}^{w_{1}} \otimes \alpha_{2}^{w_{2}} \otimes \cdots \otimes \alpha_{q}^{w_{q}} \\
& =\left\langle\prod_{i=1}^{q} \mu_{\alpha_{i}}^{w_{i}}, 1-\prod_{i=1}^{q}\left(1-v_{\alpha_{i}}\right)^{w_{i}}\right\rangle
\end{aligned}
$$

Based on above operators, evidence bodies with weights $w_{1}, w_{2} \cdots, w_{q}$ can be combined in the framework of MCDM. Two kinds of final intuitionistic fuzzy probability of $A_{i}, i=1,2, \cdots, p$, can be obtained as:

$$
\begin{aligned}
A_{i} & =I F W A\left(A_{i 1}, A_{i 2}, \cdots, A_{i q}\right) \\
& =\bigoplus_{j=1}^{q} w_{j} A_{i j} \\
& =w_{1} A_{i 1} \oplus w_{2} A_{i 2} \oplus \cdots \oplus w_{q} A_{i q} \\
& =\left\langle 1-\prod_{j=1}^{q}\left(1-\mu_{j}\left(A_{i}\right)\right)^{w_{j}}, \prod_{j=1}^{q} v_{j}\left(A_{i}\right)^{w_{j}}\right\rangle \\
A_{i} & =I F W G\left(A_{i 1}, A_{i 2}, \cdots, A_{i q}\right) \\
& =\bigotimes_{j=1}^{q}\left(A_{i j}\right)^{w_{j}} \\
& =\left(A_{i 1}\right)^{w_{1}} \otimes\left(A_{i 2}\right)^{w_{2}} \otimes \cdots \otimes\left(A_{i q}\right)^{w_{q}} \\
& =\left\langle\prod_{j=1}^{q} \mu_{j}\left(A_{i}\right)^{w_{j}}, 1-\prod_{j=1}^{q}\left(1-v_{j}\left(A_{i}\right)\right)^{w_{j}}\right\rangle
\end{aligned}
$$

At last, by ranking the intuitionistic fuzzy values $A_{1}, A_{2}, \cdots, A_{p}$, we can make a decision.

In the above aggregation operators, the weights are all real numbers in $[0,1]$. While sometimes, in the evidence combination problem, the weights of evidence sources are represented with IFVs, which are essentially interval values. Let $w_{j}=<\eta_{j}, \rho_{j}>, j=1,2, \cdots, q$, be the weights of evidence sources. Based on the equivalence between IFVs and interval values, they can be rewritten as $w_{j}=\left[w_{j}^{L}, w_{j}^{U}\right]=\left[\eta_{j}, 1-\rho_{j}\right]$, $j=1,2, \cdots, q$. Wang and Elhag [52] have defined the normalization conditions for interval valued weights as:

Definition 3.1 [52]. If an interval weight vector $\boldsymbol{w}=\left(w_{1}, w_{2} \cdots, w_{q}\right)^{T}$ with $w_{j}=\left[w_{j}^{L}, w_{j}^{U}\right]$ and $0 \leq w_{j}^{L} \leq w_{j}^{U} \leq 1$ for $j=1,2, \cdots, q$ satisfies the following two conditions:

$$
\sum_{j=1}^{q} w_{j}^{U}-\left(w_{k}^{U}-w_{k}^{L}\right) \geq 1, \forall k \in\{1, \cdots, q\}
$$




$$
\sum_{j=1}^{q} w_{j}^{L}+\left(w_{k}^{U}-w_{k}^{L}\right) \leq 1, \forall k \in\{1, \cdots, q\}
$$

then it is normalized, otherwise it is not normalized.

Motivated by this definition, we can obtain normalization conditions for intuitionistic fuzzy weights.

Theorem 3.1. An intuitionistic fuzzy weight vector $\boldsymbol{w}=\left(w_{1}, w_{2} \cdots, w_{q}\right)^{T}$ with $w_{j}=<\eta_{j}, \rho_{j}>$ for $j=1,2, \cdots, q$ is said to be normalized if and only if it satisfies the following two conditions:

$$
\begin{aligned}
& \sum_{j=1}^{q}\left(1-\rho_{j}\right)-\pi_{k} \geq 1, \forall k \in\{1, \cdots, q\} \\
& \sum_{j=1}^{q} \eta_{j}+\pi_{k} \leq 1, \forall k \in\{1, \cdots, q\}
\end{aligned}
$$

where $\pi_{k}=1-\eta_{k}-\rho_{k}$ for $k=1,2, \cdots, q$.

Proof. It is straightforward if we replace $w_{j}^{U}, w_{j}^{L}$, and $\left(w_{k}^{U}-w_{k}^{L}\right)$ in Eqs. (23) and (24) with $1-\rho_{j}, \eta_{j}$, and $\pi_{k}$, respectively.

New intuitionistic fuzzy aggregation operators have been developed in [47] to address MCDM problem with intuitionistic fuzzy weights. The developed new intuitionistic fuzzy aggregation operators are defined based on the following operations.

Definition 3.2 [47]. Let $A=\{\mu, v\}$ and $w=\langle\eta, \rho\rangle$ be two intuitionistic fuzzy values. Then the following operation rule can be defined:

$$
w A=\left\{1-(1-\mu)^{\eta}, v^{1-\rho}\right\}, A^{w}=\left\{\mu^{1-\rho}, 1-(1-v)^{\eta}\right\} .
$$

Note that in the above definition, if $\eta=1-\rho=k$, then the IFV $w$ can be expressed by an interval $[k, k]$, which indicates that $w$ degenerates to real number $k$. In this case, we have

$$
\begin{aligned}
& w A=\left\langle 1-(1-\mu)^{k}, v^{k}\right\rangle=k A \\
& A^{w}=\left\langle\mu^{k}, 1-(1-v)^{k}\right\rangle=A^{k}
\end{aligned}
$$

In such case, we have $w A=k A$ and $A^{w}=A^{k}$. So operation $w A$ can be regard as an extension of operation $k A$, operation $A^{w}$ can be regard as an extension of operation $A^{k}$. Moreover, given $\mathbf{0}=\langle 0,1\rangle$ and $\mathbf{1}=\langle 1,0\rangle$, for each IFV $A$, we have:

(1) $\mathbf{0} A=\mathbf{0}, \mathbf{1} A=1 A=A$,

(2) $A^{0}=1, A^{1}=A^{1}=A$.

Definition 3.3 [47]. Let $\alpha_{i}=\left\langle\mu_{\alpha_{i}}, v_{\alpha_{i}}\right\rangle \quad(i=1,2, \cdots, q)$ be a collection of intuitionistic fuzzy values, $\boldsymbol{w}=\left(w_{1}, w_{2}, \cdots, w_{q}\right)^{T}$ be the intuitionistic fuzzy weights vector such that $w_{i}=\left\langle\eta_{i}, \rho_{i}\right\rangle$ for $i=1,2, \cdots, q$. Then the generalized intuitionistic fuzzy weighted averaging operator (G-IFWA) and generalized intuitionistic fuzzy weighted geometric operator (G-IFWG) can be defined as follows: 


$$
\begin{aligned}
& G-I F W A\left(\alpha_{1}, \alpha_{2}, \cdots, \alpha_{q}\right) \\
& =\bigoplus_{i=1}^{q} w_{i} \alpha_{i}=w_{1} \alpha_{1} \oplus w_{2} \alpha_{2} \oplus \cdots \oplus w_{q} \alpha_{q} \\
& =\left\langle 1-\prod_{i=1}^{q}\left(1-\mu_{\alpha_{i}}\right)^{\eta_{i}}, \prod_{i=1}^{q} v_{\alpha_{i}}^{1-\rho_{i}}\right\rangle \\
& G-I F W G\left(\alpha_{1}, \alpha_{2}, \cdots, \alpha_{q}\right) \\
& =\bigotimes_{i=1}^{q}\left(\alpha_{i}^{w_{i}}\right)=\alpha_{1}^{w_{1}} \otimes \alpha_{2}^{w_{2}} \otimes \cdots \otimes \alpha_{q}^{w_{q}} \\
& =\left\langle\prod_{i=1}^{q} \mu_{\alpha_{i}}^{1-\rho_{i}}, 1-\prod_{i=1}^{q}\left(1-v_{\alpha_{i}}\right)^{\eta_{i}}\right\rangle
\end{aligned}
$$

For evidence sources with intuitionistic fuzzy weights $w_{j}=\left\langle\eta_{j}, \rho_{j}\right\rangle, j=1,2, \cdots, q$, they can be combined based on G-IFWA and G-IFWG operators in the framework of MCDM. Thus, the final intuitionistic fuzzy probability of $A_{i}, i=1,2, \cdots, p$, can be obtained as:

$$
\begin{aligned}
A_{i} & =G-I F W A\left(A_{i 1}, A_{i 2}, \cdots, A_{i q}\right) \\
& =\bigoplus_{j=1}^{q} w_{j} A_{i j} \\
& =w_{1} A_{i 1} \oplus w_{2} A_{i 2} \oplus \cdots \oplus w_{q} A_{i q} \\
& =\left\langle 1-\prod_{j=1}^{q}\left(1-\mu_{j}\left(A_{i}\right)\right)^{\eta_{j}}, \prod_{j=1}^{q} v_{j}\left(A_{i}\right)^{1-\rho_{j}}\right\rangle \\
A_{i} & =G-I F W G\left(A_{i 1}, A_{i 2}, \cdots, A_{i q}\right) \\
& =\bigotimes_{j=1}^{q}\left(A_{i j}\right)^{w_{j}} \\
& =\left(A_{i 1}\right)^{w_{1}} \otimes\left(A_{i 2}\right)^{w_{2}} \otimes \cdots \otimes\left(A_{i q}\right)^{w_{q}} \\
& =\left\langle\prod_{j=1}^{q} \mu_{j}\left(A_{i}\right)^{1-\rho_{j}}, 1-\prod_{j=1}^{q}\left(1-v_{j}\left(A_{i}\right)\right)^{\eta_{j}}\right\rangle
\end{aligned}
$$

Then, by ranking the intuitionistic fuzzy values $A_{1}, A_{2}, \cdots, A_{p}$, final decision can be made.

\subsection{Evidence combination based on evidence discounting operation}

\subsubsection{Generalized evidence discounting operation}

Let $\Omega=\left\{A_{1}, A_{2}, \cdots, A_{p}\right\}$ be the discernment frame. $m$ is a belief structure on $\Omega .\left\langle\mu\left(A_{i}\right), v\left(A_{i}\right)\right\rangle$ $(i=1,2, \cdots, p)$ is the intuitionistic fuzzy probability associated with $m$, where $\mu\left(A_{i}\right)=\operatorname{Bel}\left(A_{i}\right)$, $v\left(A_{i}\right)=1-P l\left(A_{i}\right)$. Considering the definition of belief function and plausibility function shown as Eqs. (3) and (4), we can get:

$$
\begin{gathered}
\mu\left(A_{i}\right)=\operatorname{Bel}\left(A_{i}\right)=\sum_{B \subseteq A_{i}} m(B)=m\left(A_{i}\right) \\
v\left(A_{i}\right)=1-P l\left(A_{i}\right)=\sum_{B \cap A_{i}=\varnothing} m(B)
\end{gathered}
$$

Theorem 3.2. Let $\Omega=\left\{A_{1}, A_{2}, \cdots, A_{p}\right\}$ be the discernment frame. Suppose that the reliability degree of $m$ is $\lambda, \lambda \in[0,1] . m^{\prime}$ is a belief structure obtained by discounting $m$ shown in Eq. (11). $\left\langle\mu\left(A_{i}\right), v\left(A_{i}\right)\right\rangle$ and $\left\langle\mu^{\prime}\left(A_{i}\right), v^{\prime}\left(A_{i}\right)\right\rangle, \quad i=1,2, \cdots, p$, are intuitionistic fuzzy probabilities generated by $m$ and $m^{\prime}$, respectively. Then, $\mu^{\prime}\left(A_{i}\right)=\lambda \cdot \mu\left(A_{i}\right), v^{\prime}\left(A_{i}\right)=\lambda \cdot v\left(A_{i}\right)$. 
Proof. Given Shafer's discounting operation in Eq. (11), we have: $m^{\prime}(A)=\lambda m(A)$ for $A \subset \Omega$ and $m^{\prime}(\Omega)=1-\lambda+\lambda m(\Omega)$.

Considering Eqs. (33) and (34), we can further get:

$m^{\prime}\left(A_{i}\right)=\lambda m\left(A_{i}\right)=\lambda \mu\left(A_{i}\right), \quad i=1,2, \cdots, p$.

Since $\Omega \cap A_{i} \neq \varnothing$, we have $\sum_{B \cap A_{i}=\varnothing} m^{\prime}(B)=\lambda \cdot \sum_{B \cap A_{i}=\varnothing} m(B)=\lambda \cdot v\left(A_{i}\right)$.

Consequently,

$$
\mu^{\prime}\left(A_{i}\right)=m^{\prime}\left(A_{i}\right)=\lambda \cdot \mu\left(A_{i}\right), \quad v^{\prime}\left(A_{i}\right)=\sum_{B \cap A_{i}=\varnothing} m^{\prime}(B)=\lambda \cdot v\left(A_{i}\right) .
$$

Theorem 3.2 presents the expression of discounting operation in the framework of intuitionistic fuzzy MCDM. From the point of view of interval values, the real number $\lambda$ can be expressed by interval $[\lambda, \lambda]$, which can be further written as intuitionistic fuzzy value $\langle\lambda, 1-\lambda\rangle$. In such case we have $\mu^{\prime}\left(A_{i}\right)=\lambda \cdot \mu_{(}\left(A_{i}\right)$, $v^{\prime}\left(A_{i}\right)=(1-(1-\lambda)) \cdot v\left(A_{i}\right)$.

In the above discounting operation, the reliability factors are all real numbers in $[0,1]$. However, when evaluating the reliability of evidence sources, we may be uncertain about the reliability factors due to the limitation of knowledge at hand. So the reliability of evidence sources may be represented with intuitionistic fuzzy values. So we need to extend Shafer's discounting operation to cope with intuitionistic fuzzy reliability factors. The developed new discounting operation can be applied in the evidence combination problem when the weights are represented with intuitionistic fuzzy values. At first we define a new intuitionistic fuzzy operation:

Definition 3.4. Let $A=\{\mu, v\}$ and $\lambda=\langle\eta, \rho\rangle$ be two intuitionistic fuzzy values. Then we define a new intuitionistic fuzzy operation as: $\lambda \circ A=\langle\eta \mu,(1-\rho) v\rangle$.

It is clear that $0 \leq \eta \mu \leq 1,0 \leq(1-\rho) v \leq 1$, and $\eta \mu+(1-\rho) v \leq \mu+v \leq 1$. So $\lambda \circ A$ is an intuitionistic fuzzy value. $\eta \mu+(1-\rho) v \leq \mu+v$ also indicates that the uncertainty of $\lambda \circ A$ is not less than $A$, i.e., $\pi_{\lambda \circ A} \geq \pi_{A}$

Based on this new intuitionistic fuzzy operation, we can define a generalized evidence discounting operation as following.

Definition 3.5. Let $\Omega=\left\{A_{1}, A_{2}, \cdots, A_{p}\right\}$ be the discernment frame. Suppose that the reliability degree of $m$ is an intuitionistic fuzzy value $\lambda, \lambda=\langle\eta, \rho\rangle . m^{\prime}$ is a belief structure obtained by discounting $m$. $A_{i}=\left\langle\mu\left(A_{i}\right), v\left(A_{i}\right)\right\rangle$ and $A_{i}^{\prime}=\left\langle\mu^{\prime}\left(A_{i}\right), v^{\prime}\left(A_{i}\right)\right\rangle, i=1,2, \cdots, p$, are intuitionistic fuzzy probabilities generated by $m$ and $m^{\prime}$, respectively. Then, $A_{i}^{\prime}=\lambda \circ A_{i}$ for $i=1,2, \cdots, p$.

We can notice that in the above definition, if $\eta=1-\rho=k$, then the intuitionistic fuzzy value $\lambda$ can be expressed by an interval $[k, k]$. Thus, $\lambda$ degenerates to real number $k$. In this case, we have:

$$
\lambda \circ A=\langle k \mu, k v\rangle
$$

Eq. (35) is similar to the expression of discounting operation in Theorem 3.2. So the expression of Shafer's discounting operation in the framework of intuitionistic fuzzy MCDM can be regarded as a special case of our generalized discounting operation. Moreover, given $\mathbf{0}=\langle 0,1\rangle$ and $\mathbf{1}=\langle 1,0\rangle$, for each IFV $A$, 
we have:

$$
\begin{gathered}
\mathbf{0} \circ A=\langle 0,0\rangle \\
\mathbf{1} \circ A=A
\end{gathered}
$$

In the application of evidence combination, when the reliability of evidence is 0 , i.e., it is totally unreliable, the information provided by it is totally uncertain. In the situation where the evidence source is completely reliable, the belief function and plausibility function are unchanged after discounting. Eqs. (36) and (37) coincide with these analyses.

Theorem 3.3. Let $A=\{\mu, v\}, \lambda_{1}=\left\langle\eta_{1}, \rho_{1}\right\rangle$, and $\lambda_{2}=\left\langle\eta_{2}, \rho_{2}\right\rangle$ be three intuitionistic fuzzy values. If $\lambda_{1}>\lambda_{2}$, then the hesitancy degree of $\lambda_{1} \circ A$ is less than that of $\lambda_{2} \circ A$, i.e., $\pi_{\lambda_{1} \circ A}<\pi_{\lambda_{2} \circ A}$.

Proof. Considering the relation in Definition 2.11, we have $\lambda_{1}>\lambda_{2} \Leftrightarrow \eta_{1}>\eta_{2}, \rho_{1}<\rho_{2}$. Then it follows that: $\eta_{1} \mu>\eta_{2} \mu$ and $\left(1-\rho_{1}\right) v>\left(1-\rho_{2}\right) v$.

Consequently, $1-\left(\eta_{1} \mu+\left(1-\rho_{1}\right) v\right)<1-\left(\eta_{2} \mu+\left(1-\rho_{2}\right) v\right)$, which indicates that $\pi_{\lambda_{1} \circ A}<\pi_{\lambda_{2} \circ A}$.

It is clear that evidence discounting will bring an increase in the uncertainty, which has also been illustrated by $\pi_{\lambda \circ A} \geq \pi_{A}$. We would have in mind that greater reliability factor will cause less discounting degree on the original evidence. So less growth on uncertainty will be generated. Theorem 3.3 reflects the negative correlation between evidence reliability and the increase of uncertainty.

\subsubsection{Combination operation on IFVs}

From the point of view of belief function, we can define a combination operation on IFVs. Since an IFV $A=\left\langle\mu_{A}, v_{A}\right\rangle$ is developed from an AIFS $A=\left\{\left\langle x, \mu_{A}(x), v_{A}(x)\right\rangle\right\}$ on $X=\{x\}$, where $\mu_{A}$ and $v_{A}$ are the membership degree and non-membership degree of $x$ belong to $A$. Following this original interpretation of AIFS, in the application of MCDM, $\mu_{A}(x)$ and $v_{A}(x)$ can be regarded as the answer of "Is the alternative excellent or not for a criterion?" [53]. The hypotheses frame of the answer is $\{\{Y e s\},\{N o\},\{Y e s, N o\}\}$. In the framework of evidence theory, we have $m(Y e s)=\mu_{A}$ and $m(N o)=v_{A}$. Since $\pi_{A}$ is the hesitation degree, we can get $m(Y e s, N o)=\pi_{A}$. Therefore, an IFV $A$ can elicit a belief structure as:

$$
\left\{\begin{array}{l}
m(Y e s)=\mu_{A} \\
m(N o)=v_{A} \\
m(Y e s, N o)=\pi_{A}
\end{array}\right.
$$

Given two IFVs $A_{11}=\left\langle\mu_{1}, v_{1}\right\rangle$ and $A_{12}=\left\langle\mu_{2}, v_{2}\right\rangle$, which corresponds to the evaluation results of alternative $A_{1}$ for criteria $C_{1}$ and $C_{2}$, we can get two belief structures $m_{1}$ and $m_{2}$ as:

$$
\begin{gathered}
\left\{\begin{array}{l}
m_{1}(\text { Yes })=\mu_{1} \\
m_{1}(N o)=v_{1} \\
m_{1}(\text { Yes }, N o)=\pi_{1}
\end{array}\right. \\
\left\{\begin{array}{l}
m_{2}(\text { Yes })=\mu_{2} \\
m_{2}(N o)=v_{2} \\
m_{2}(\text { Yes }, N o)=\pi_{2}
\end{array}\right.
\end{gathered}
$$

$m_{1}$ and $m_{2}$ represent the answer to the question of "Is the alternative $A_{1}$ excellent or not for a criterion $C_{1}$ " and "Is the alternative $A_{1}$ excellent or not for a criterion $C_{2}$ ", respectively. To reach a 
comprehensive evaluation on $A_{1}$, we can combine two belief structures using Dempster's rule of combination as:

$$
m_{1} \oplus m_{2}\left(H_{1}\right)=\frac{\sum_{H_{2} \cap H_{3}=H_{1}} m_{1}\left(H_{2}\right) m_{2}\left(H_{3}\right)}{1-\sum_{H_{2} \cap H_{3}=\varnothing} m_{2}\left(H_{2}\right) m_{3}\left(H_{3}\right)}
$$

where $H_{1}, H_{2}, H_{3} \in\{\{Y e s\},\{N o\},\{Y e s, N o\}\}$.

By Eqs. (38) and (41), we can get:

$$
\left\{\begin{array}{l}
m_{1} \oplus m_{2}(\text { Yes })=\frac{\mu_{1}\left(1-v_{2}\right)+\mu_{2} \pi_{1}}{1-\mu_{1} v_{2}-\mu_{2} v_{1}} \\
m_{1} \oplus m_{2}(N o)=\frac{v_{1}\left(1-\mu_{2}\right)+v_{2} \pi_{1}}{1-\mu_{1} v_{2}-\mu_{2} v_{1}} \\
m_{1} \oplus m_{2}(\text { Yes }, N o)=\frac{\pi_{1} \pi_{2}}{1-\mu_{1} v_{2}-\mu_{2} v_{1}}
\end{array}\right.
$$

where $\mu_{1} v_{2}+\mu_{2} v_{1} \neq 1$.

Consequently, similar to the combination operation on AIFSs introduced in [56], we can also define a combination operation on IFVs as following.

Definition 3.6. Let $A_{1}=\left\langle\mu_{1}, v_{1}\right\rangle$ and $A_{2}=\left\langle\mu_{2}, v_{2}\right\rangle$ be two IFVs. A combination operation on $A_{1}$ and $A_{2}$, denoted by $A_{1} \square A_{2}$, is defined by:

$$
A_{1} \square A_{2}=\left\langle\frac{\mu_{1}\left(1-v_{2}\right)+\mu_{2} \pi_{1}}{1-\mu_{1} v_{2}-\mu_{2} v_{1}}, \frac{v_{1}\left(1-\mu_{2}\right)+v_{2} \pi_{1}}{1-\mu_{1} v_{2}-\mu_{2} v_{1}}\right\rangle
$$

It is obvious that $A_{1} \square A_{2}$ is also an IFV. By Eq. (42), we can get the hesitancy degree of $A_{1} \square A_{2}$ as:

$$
\pi_{A_{1} \square A_{2}}=\frac{\pi_{1} \pi_{2}}{1-\mu_{1} v_{2}-\mu_{2} v_{1}}
$$

Since the Dempster's rule can be applied to combine more than two belief structures, the operation $\square$ can be extended to combine $N$ IFVs, denoted as $\square{ }_{j=1}^{N} A_{j}$. Considering the commutativity and associativity of Dempster's combination rule, we can get following properties:

(1) (Commutativity) $A_{1} \square A_{2}=A_{2} \square A_{1}$

(2) (Associativity) $A_{1} \square A_{2} \square A_{3}=\left(A_{1} \square A_{2}\right) \square A_{3}=A_{1} \square\left(A_{2} \square A_{3}\right)$

Therefore, the combination of $N$ IFVs is independent of the order of IFVs.

\subsubsection{Combine unreliable evidence sources based on evidence discounting}

For the MCDM model in Eq. (18), which is corresponding to an evidence combination problem, let the reliability factor of each evidence source be $\lambda_{j}=\left\langle\eta_{j}, \rho_{j}\right\rangle, j=1,2, \cdots, q$. Then evidence bodies can be discounted by the generalized discounting operation as: 


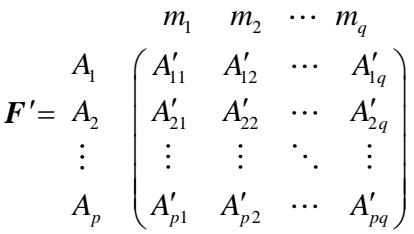

$$
\begin{aligned}
& \begin{array}{llll}
m_{1} & m_{2} & \cdots & m_{q}
\end{array}
\end{aligned}
$$

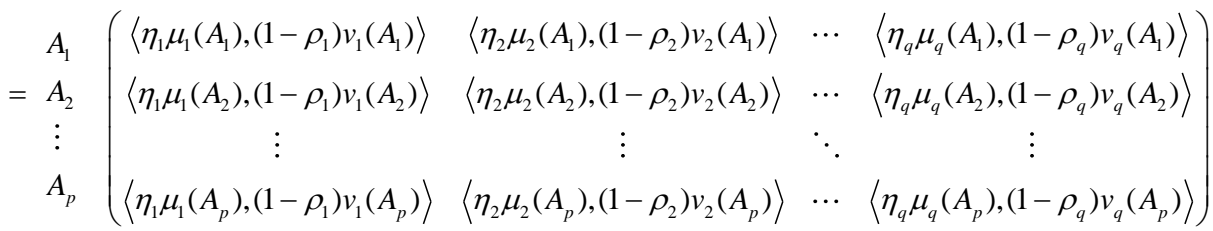

In the framework of MCDM, the evaluation results of each alternative for all criteria can be combined based on the combination operation $\square$ on multi intuitionistic fuzzy values. So the final probability of $A_{i}$, $i=1,2, \cdots, p$ can be obtained as:

$$
\begin{aligned}
& A_{i}=\square_{j=1}^{q} A_{i j}^{\prime}=\square_{j=1}^{q}\left(\lambda_{1} \circ A_{i 1}, \lambda_{2} \circ A_{i 2}, \cdots, \lambda_{q} \circ A_{i q}\right) \\
& =\left\langle\eta_{1} \mu_{1}\left(A_{i}\right),\left(1-\rho_{1}\right) v_{1}\left(A_{i}\right)\right\rangle \square \cdots \square\left\langle\eta_{q} \mu_{q}\left(A_{i}\right),\left(1-\rho_{q}\right) v_{q}\left(A_{i}\right)\right\rangle
\end{aligned}
$$

At last, we can get the fusion result by ranking intuitionistic fuzzy values $A_{1}, A_{2}, \cdots, A_{p}$.

\section{Dynamic reliability evaluation based on MCDM model}

In above section, when combining unreliable evidence sources, the terminologies "evidence weight" and "evidence reliability" are applied. As discussed in [54], there is clear difference between evidence weight (importance) and evidence reliability. In information fusion, for example, the importance of an information source is indicated by weight as granted to the source by a fusion system designer, which can be relative to other information sources; the reliability of an information source represents its ability to provide correct assessment or solution for a given problem [55]. In other words, the reliability and importance of a piece of evidence may not measure the same property of the evidence, and as such they need to be treated separately in an inference process. In this paper, we treat the weight of evidence as its dynamic reliability, while the reliability is regarded as the static reliability determined by the performance of sensors. Moreover, we make an assumption that all combination rules in the framework of MCDM are suitable for both normalized static and dynamic reliability factors.

Now that aforementioned combination rules can deal with IFV weights /reliability factors, is it necessary to evaluate the precise dynamic reliability of evidence bodies? The answer is positive. Since the static reliability is related to the property of sensors or the knowledge of experts, it can be only evaluated based on a priori knowledge; and the static reliability is usually uncertain and impression. We need to evaluate dynamic reliability factor precisely in order to keep the uncertainty of final reliability factor in a low level. In addition, there are many methods addressing the evaluation of static reliability. So this problem will be out of the scope of this paper, and we would like to evaluate the dynamic reliability of evidence sources based on MCDM in intuitionistic fuzzy environment. Nevertheless, we must keep in mind that evidence reliability consists of two parts, i.e., static reliability and dynamic reliability.

By transforming evidence combination to MCDM model in intuitionistic fuzzy environment, the dynamic reliability of evidence source is corresponding to the weight of criterion. So we can evaluate the dynamic reliability of evidence sources using criteria weighting approaches. Although many researchers [57]-[59] have been dedicated to developing methods of determining criteria weights in MCDM, most of them are based on the ordered weighting averaging (OWA) operation and non-linear optimization. Moreover, the so-called ideal solutions are needed for some approaches to minimize the instantaneous errors. We notice 
that these methods may be not applicable in the evaluation of evidence reliability, which concerns more about timeliness without enough a priori knowledge. So we will propose a new reliability evaluation approach based on probabilistic comparison between IFVs.

\subsection{Probabilistic comparison between IFVs}

In Definition 2.11, the ordinary partial ordered relations between two IFVs $A=\left\langle\mu_{A}, v_{A}\right\rangle$ and $B=\left\langle\mu_{B}, v_{B}\right\rangle$ have been defined as $A \geq B \Leftrightarrow \mu_{A} \geq \mu_{B}, v_{A} \leq v_{B}$. But for the situation of $\mu_{A} \geq \mu_{B}, v_{A} \geq v_{B}$, we cannot give their order. In addition, the ranking methods based on score function and accuracy function can provide the qualitative order between IFVs, which is less applicable than quantitative comparison between IFVs. Considering the equivalence between IFVs and interval values, we can probabilistically compare IFVs based on the comparison between interval values.

Definition 4.1 [60][61]. Let $\alpha=\left[a^{L}, a^{U}\right]$ and $\beta=\left[b^{L}, b^{U}\right]$ be two interval values such that $a^{L}<a^{U}$ and $b^{L}<b^{U}$. The possible degree of the interval value $\alpha$ preceding the interval value $\beta$, denoted as $P_{(\alpha \geq \beta)}$, is defined as:

$$
P_{(\alpha \geq \beta)}=\min \left\{1, \max \left\{\frac{a^{U}-b^{L}}{\left(a^{U}-a^{L}\right)+\left(b^{U}-b^{L}\right)}, 0\right\}\right\}
$$

It is worth mentioning that if $\alpha$ and $\beta$ both reduce to precise values, i.e., $a^{L}=a^{U}=a, b^{L}=b^{U}=b$, Eq. (47) is undefined owing to the $\left(a^{U}-a^{L}\right)+\left(b^{U}-b^{L}\right)=0$. In this special case, we take $P_{(\alpha \geq \beta)}=1$ for $a>b$. Particularly, we assume $P_{(\alpha \geq \beta)}=0.5$ for $a=b$.

Theorem $4.1[61][62]$. For two interval values $\alpha=\left[a^{L}, a^{U}\right]$ and $\beta=\left[b^{L}, b^{U}\right]$, the possible degree $P_{(\alpha \geq \beta)}$ satisfies the following properties:

(1) $0 \leq P_{(\alpha \geq \beta)} \leq 1$;

(2) $P_{(\alpha \geq \alpha)}=0.5$;

(3) $P_{(\alpha \geq \beta)}=1 \Leftrightarrow a^{L} \geq b^{U}$;

(4) $P_{(\alpha \geq \beta)}+P_{(\beta \geq \alpha)}=1$.

Proof. Properties (1) and (2) are both straightforward from the definition of $P_{(\alpha \geq \beta)}$.

(3) $P_{(\alpha \geq \beta)}=1 \Rightarrow \max \left\{\frac{a^{U}-b^{L}}{\left(a^{U}-a^{L}\right)+\left(b^{U}-b^{L}\right)}, 0\right\} \geq 1 \Rightarrow \frac{a^{U}-b^{L}}{\left(a^{U}-a^{L}\right)+\left(b^{U}-b^{L}\right)} \geq 1$.

Since the denominator $\left(a^{U}-a^{L}\right)+\left(b^{U}-b^{L}\right)$ is positive, we can get $a^{U}-b^{L}>0$. Then it follows that: $a^{U}-b^{L} \geq\left(a^{U}-a^{L}\right)+\left(b^{U}-b^{L}\right)=a^{U}-b^{L}-\left(a^{L}-b^{U}\right)$, which indicates that $a^{L}-b^{U} \geq 0$, i.e., $a^{L} \geq b^{U}$.

The inverse proof is trivial.

(4) Three cases listed in Fig.1 need to be considered.

(i) For the separation case, we get $a^{L} \geq b^{U}, P_{(\alpha \geq \beta)}=1$ and $P_{(\beta \geq \alpha)}=0$. Thus $P_{(\alpha \geq \beta)}+P_{(\beta \geq \alpha)}=1$.

(ii) The overlapping case indicates that $b^{L} \leq a^{L} \leq b^{U} \leq a^{U}$. Then we have:

$$
P_{(\alpha \geq \beta)}=\frac{a^{U}-b^{L}}{\left(a^{U}-a^{L}\right)+\left(b^{U}-b^{L}\right)}, \quad P_{(\beta \geq \alpha)}=\frac{b^{U}-a^{L}}{\left(a^{U}-a^{L}\right)+\left(b^{U}-b^{L}\right)} .
$$

Thus, 
$P_{(\alpha \geq \beta)}+P_{(\beta \geq \alpha)}=\frac{a^{U}-b^{L}}{\left(a^{U}-a^{L}\right)+\left(b^{U}-b^{L}\right)}+\frac{b^{U}-a^{L}}{\left(a^{U}-a^{L}\right)+\left(b^{U}-b^{L}\right)}=1$.

(iii) In the inclusion case, we notice that $b^{L} \geq a^{L}, b^{U} \leq a^{U}$. Then we get:

$P_{(\alpha \geq \beta)}=\frac{a^{U}-b^{L}}{\left(a^{U}-a^{L}\right)+\left(b^{U}-b^{L}\right)}, \quad P_{(\beta \geq \alpha)}=\frac{b^{U}-a^{L}}{\left(a^{U}-a^{L}\right)+\left(b^{U}-b^{L}\right)}$.

Therefore,

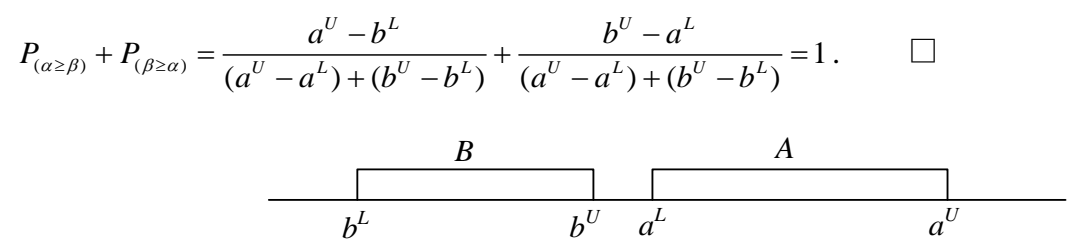

(i) Separation case

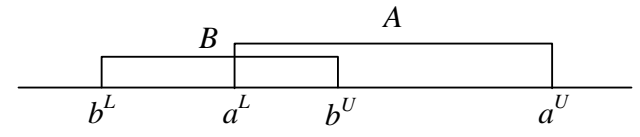

(ii) Overlapping case

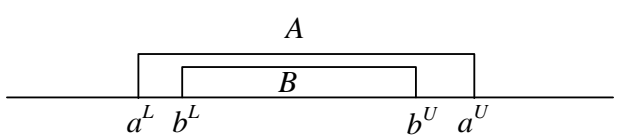

(iii) Inclusion case

Fig.1. Cases of interval relations

Since IFVs $A=\left\langle\mu_{A}, v_{A}\right\rangle$ and $B=\left\langle\mu_{B}, v_{B}\right\rangle$ can be expressed by interval values $A=\left[\mu_{A}, 1-v_{A}\right]$ and $B=\left[\mu_{B}, 1-v_{B}\right]$, respectively, we can also define the possibility of $A \geq B$ form Definition 4.1.

Definition 4.2. Let $A=\left\langle\mu_{A}, v_{A}\right\rangle$ and $B=\left\langle\mu_{B}, v_{B}\right\rangle$ be two IFVs with $\pi_{A} \cdot \pi_{B} \neq 0$. The possible degree of $A \geq B$ can defined as:

$$
P_{(A \geq B)}=\min \left\{1, \max \left\{\frac{1-v_{A}-\mu_{B}}{\pi_{A}+\pi_{B}}, 0\right\}\right\}
$$

In the case of $\pi_{A}+\pi_{B}=0, A$ and $B$ are both associated with precise real numbers. So we have $P_{(A \geq B)}=1$ if $\mu_{A}>\mu_{B}$ and $P_{(A \geq B)}=0.5$ if $\mu_{A}=\mu_{B}$.

Theorem 4.2. Given two IFVs $A=\left\langle\mu_{A}, v_{A}\right\rangle$ and $B=\left[\mu_{B}, 1-v_{B}\right]$, we have:

(1) $0 \leq P_{(A \geq B)} \leq 1$;

(2) $P_{(A \geq A)}=0.5$;

(3) $P_{(A \geq B)}=1 \Leftrightarrow \mu_{A} \geq 1-v_{B}$;

(4) $P_{(A \geq B)}+P_{(B \geq A)}=1$.

Proof. They are all straightforward from Theorem 4.1 and Definition 4.2.

It is worth noticing that $P_{(A \geq B)}=1 \Rightarrow 1-v_{A} \geq \mu_{A} \geq 1-v_{B} \geq \mu_{B} \Rightarrow \mu_{A} \geq \mu_{B}, v_{A} \leq v_{B}$ can be obtained from the third property in Theorem 4.2, but not vice versa. This is different from the ordinary partial ordered relation defined in Definition 2.11. So the relation $A \geq B$ defined in Definition 4.2 is much stricter than ordinary partial ordered relation in Definition 2.11.

We now suppose that there are $n$ input arguments IFVs $A_{1}, A_{2}, \cdots, A_{n}$ with the forms of $A_{i}=\left\langle\mu_{A_{i}}, v_{A_{i}}\right\rangle$, 
$i=1,2, \cdots, n$. To rank these arguments, we first compare each argument $A_{i}$ with all arguments $A_{j}$, $i, j=1,2, \cdots, n$. By Definition 4.2, we have:

$$
P_{\left(A_{i} \geq A_{j}\right)}=\min \left\{1, \max \left\{\frac{1-v_{A_{i}}-\mu_{A_{j}}}{\pi_{A_{i}}+\pi_{A_{j}}}, 0\right\}\right\}, \quad i, j=1,2, \cdots, n
$$

For simplicity, we let $P_{i j}=P_{\left(A_{i} \geq A_{j}\right)}$. Then we can construct a complementary comparison matrix as follows:

$$
\boldsymbol{P}=\left[\begin{array}{cccc}
P_{11} & P_{12} & \cdots & P_{1 n} \\
P_{21} & P_{22} & \cdots & P_{2 n} \\
\vdots & \vdots & \ddots & \vdots \\
P_{n 1} & P_{n 2} & \cdots & P_{n n}
\end{array}\right]
$$

Obviously, $\forall i, j \in\{1,2, \cdots, n\}$, we have $0 \leq P_{i j} \leq 1, \quad P_{i j}+P_{j i}=1$, and $P_{i i}=0.5$.

Summing all elements in each row of matrix $\boldsymbol{P}$, we have:

$$
P_{i}=\sum_{j=1}^{n} P_{i j}
$$

Then we can rank the arguments $A_{1}, A_{2}, \cdots, A_{n}$ in accordance with the value of $P_{i}, \quad i \in\{1,2, \cdots, n\}$.

To make this ranking method more transparent and comparable with ranking methods proposed in Definition 2.14, we reconsider Example 2.2.

$$
A_{1}=\langle 0.6,0.3\rangle, \quad A_{2}=\langle 0.45,0.2\rangle, \quad A_{3}=\langle 0.5,0.25\rangle
$$

By Definition 4.2, we get complementary matrix:

$$
\boldsymbol{P}=\left[\begin{array}{lll}
0.50 & 0.56 & 0.57 \\
0.44 & 0.50 & 0.50 \\
0.43 & 0.50 & 0.50
\end{array}\right]
$$

Then,

$P_{1}=0.50+0.56+0.57=1.63, \quad P_{2}=0.44+0.50+0.50=1.44, \quad P_{3}=0.43+0.50+0.50=1.43$.

So the ranking order is $A_{1} \succ A_{2} \succ A_{3}$.

We can see that this result is not identical to that from the ranking method in Definition 2.14. If we take $P_{i}=\sum_{j=1}^{n} P_{i j}$ as a special score function, it is much more convenient for ranking IFVs than the score function $s\left(A_{i}\right)$ and the accuracy function $a\left(A_{i}\right)$. Moreover, since $s\left(A_{i}\right)$ and $a\left(A_{i}\right)$ are used separately, we cannot get a comprehensive evaluation on IFVs. So $P_{i}$ is more applicable in ranking IFVs quantitatively.

\subsection{Evaluate criteria weights based on self-assessment}

In the application of MCDM, we can get the evaluation of an alternative for each criterion. If we make an assumption of economic person, each alternative can be treated as an economic person. Rationality, self-benefit and pursuit the biggest interest are the major features of an economic person. Based on this assumption, when an economic person (alternative) is evaluated by different criteria, it would assign the greatest weight to the criterion providing the maximum support for it. In light of this observation, a perfect competition can be achieved naturally, where the largest weight is assigned as 1 , while others are 0 . However, this assignment may cause a tremendous loss of information, which is unfavorable to the ranking of all alternatives. So we need to evaluate criteria weights in an imperfect competition environment to make the best use of the superiority information as well as other information. This leaves us with the question of how 
to determine the weighs in an imperfect competition environment. It is natural and reasonable to use the probabilistic comparison between IFVs, which may be beneficial to construct an imperfect competition environment.

Let $\left\{A_{1}, A_{2}, \cdots, A_{p}\right\}$ be an alternative set to be assessed across a set of criteria $\left\{C_{1}, C_{2}, \cdots, C_{q}\right\}$. The evaluation of the alternative $A_{i}$ over criterion $C_{j}$ by IFVs is expressed as:

$$
A_{i j}=\left\langle\mu_{j}\left(A_{i}\right), v_{j}\left(A_{i}\right)\right\rangle, \quad i=1,2, \cdots, p, \quad j=1,2, \cdots, q .
$$

Then the criteria weights can be assessed by alternative $A_{i}, i=1,2, \cdots, p$, through the following three steps.

Step 1. By Definition 4.2 and Eq. (50), construct a complementary matrix $\boldsymbol{P}^{(i)}=\left[P_{m n}\right]_{q \times q}$ based on the evaluation results of an alternative $A_{i}$ over all criteria.

Step 2. Sum all elements in each row of matrix $\boldsymbol{P}^{(i)}$ by Eq. (51):

$$
P_{m}^{(i)}=\sum_{n=1}^{q} P_{m n}, m=1,2, \cdots, q
$$

Step 3. Calculate the weight of each criterion. The weight of $C_{j}$ is determined by:

$$
w_{j}^{(i)}=\frac{P_{j}^{(i)}}{\sum_{m=1}^{q} P_{m}^{(i)}}
$$

The weighting vector of all criteria based on alternative $A_{i}$ can be obtained as:

$$
\boldsymbol{w}^{(i)}=\left(w_{1}^{(i)}, w_{2}^{(i)}, \cdots, w_{q}^{(i)}\right)^{T}
$$

Since the weights of all criteria are evaluated by the assessment of each alternative, we call this method as self-assessment. We can note that the weighting vector obtained by different alternatives may be different. So we need to determine the final weighting vector. This is similar to the problem of group decision making, where a group of experts provide their preferences over criteria with $w^{(i)}$. Here we assume that the ideal weighting vector should have the least distance to all $\boldsymbol{w}^{(i)}, i=1,2, \cdots, p$. If we let $\boldsymbol{W}=\left[\boldsymbol{w}^{(1)}, \boldsymbol{w}^{(2)}, \cdots, \boldsymbol{w}^{(p)}\right]$, the Perron-Frobenius vector (the eigenvector associated to the maximal positive eigen value) of $\boldsymbol{W} \boldsymbol{W}^{T}$ can be used as the ideal weighting vector $\boldsymbol{w}$, i.e., $\boldsymbol{W} \boldsymbol{W}^{T} \cdot \boldsymbol{w}=\lambda_{\max } \boldsymbol{w}$. Usually, $\boldsymbol{w}$ need to be normalized by:

$$
\boldsymbol{w}^{\prime}=\boldsymbol{w} / \sum_{j=1}^{q} w_{j}
$$

In the light of the correspondence between dynamic reliability of evidence sources and criteria weights, we can evaluate the dynamic reliability of evidence bodies by self-assignment-based method mentioned above.

Now we give an example to illustrate the given dynamic reliability evaluation for evidence bodies.

Example 4.1. Consider a situation in which we have five belief structures $m_{1}, m_{2}, \cdots, m_{5}$ over the same frame of discernment $\Omega=\left\{A_{1}, A_{2}, A_{3}, A_{4}\right\}$. These five belief structures are listed as follows.

$$
\begin{aligned}
& m_{1}: m_{1}\left(A_{1}\right)=0.2, m_{1}\left(A_{2}\right)=0.3, m_{1}\left(A_{3}\right)=0.15, m_{1}\left(A_{4}\right)=0.15, m_{1}\left(A_{1} A_{2}\right)=0.1, m_{1}\left(A_{2} A_{3} A_{4}\right)=0.1 ; \\
& m_{2}: m_{2}\left(A_{1}\right)=0.15, m_{2}\left(A_{2}\right)=0.25, m_{2}\left(A_{3}\right)=0.15, m_{2}\left(A_{4}\right)=0.1, m_{2}\left(A_{1} A_{3} A_{4}\right)=0.15, m_{2}\left(A_{2} A_{4}\right)=0.2 ; \\
& m_{3}: m_{3}\left(A_{1}\right)=0.3, m_{3}\left(A_{2}\right)=0.35, m_{3}\left(A_{3}\right)=0.15, m_{3}\left(A_{4}\right)=0.1, m_{3}\left(A_{1} A_{2} A_{3} A_{4}\right)=0.1 ; \\
& m_{4}: m_{4}\left(A_{1}\right)=0.15, m_{4}\left(A_{2}\right)=0.3, m_{4}\left(A_{3}\right)=0.2, m_{4}\left(A_{1} A_{2} A_{3}\right)=0.15, m_{4}\left(A_{3} A_{4}\right)=0.15 ; \\
& m_{5}: m_{5}\left(A_{1}\right)=0.3, m_{5}\left(A_{2}\right)=0.2, m_{5}\left(A_{3}\right)=0.1, m_{5}\left(A_{4}\right)=0.1, m_{5}\left(A_{2} A_{3}\right)=0.2, m_{5}\left(A_{1} A_{3} A_{4}\right)=0.1 .
\end{aligned}
$$

We can get the intuitionistic fuzzy MCDM model as: 


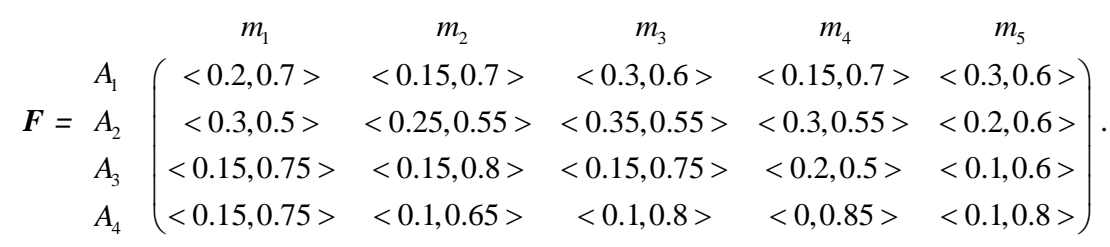

By Eqs. (49) and (50), we can get comparison matrices as following:

$$
\begin{aligned}
\boldsymbol{P}^{(1)} & =\left[\begin{array}{ccccc}
0.5 & 0.6 & 0 & 0.6 & 0 \\
0.4 & 0.5 & 0 & 0.5 & 0 \\
1 & 1 & 0.5 & 1 & 0.5 \\
0.4 & 0.5 & 0 & 0.5 & 0 \\
1 & 1 & 0.5 & 1 & 0.5
\end{array}\right], \boldsymbol{P}^{(2)}=\left[\begin{array}{ccccc}
0.5 & 0.625 & 0.5 & 0.571 & 0.75 \\
0.375 & 0.5 & 0.333 & 0.429 & 0.625 \\
0.5 & 0.667 & 0.5 & 0.6 & 0.833 \\
0.429 & 0.571 & 0.4 & 0.5 & 0.714 \\
0.25 & 0.375 & 0.167 & 0.286 & 0.5
\end{array}\right], \\
\boldsymbol{P}^{(3)} & =\left[\begin{array}{ccccc}
0.5 & 0.667 & 0.5 & 0.125 & 0.375 \\
0.333 & 0.5 & 0.333 & 0 & 0.286 \\
0.5 & 0.667 & 0.5 & 0.125 & 0.375 \\
0.875 & 1 & 0.875 & 0.5 & 0.667 \\
0.625 & 0.714 & 0.625 & 0.333 & 0.5
\end{array}\right], \boldsymbol{P}^{(4)}=\left[\begin{array}{ccccc}
0.5 & 0.429 & 0.75 & 1 & 0.75 \\
0.571 & 0.5 & 0.714 & 0.875 & 0.714 \\
0.25 & 0.286 & 0.5 & 0.8 & 0.833 \\
0 & 0.125 & 0.2 & 0.5 & 0.2 \\
0.25 & 0.286 & 0.5 & 0.8 & 0.5
\end{array}\right] .
\end{aligned}
$$

By Eq. (52), we can get:

$$
\begin{aligned}
& P_{1}^{(1)}=1.7, P_{2}^{(1)}=1.4, P_{3}^{(1)}=4, P_{4}^{(1)}=1.4, P_{5}^{(1)}=4 ; \\
& P_{1}^{(2)}=2.946, P_{2}^{(2)}=2.262, P_{3}^{(2)}=3.1, P_{4}^{(2)}=2.614, P_{5}^{(2)}=1.577 ; \\
& P_{1}^{(3)}=2.167, P_{2}^{(3)}=1.452, P_{3}^{(3)}=2.167, P_{4}^{(3)}=3.917, P_{5}^{(3)}=2.798 ; \\
& P_{1}^{(4)}=3.429, P_{2}^{(4)}=3.375, P_{3}^{(4)}=2.336, P_{4}^{(4)}=1.025, P_{5}^{(4)}=2.336 .
\end{aligned}
$$

By Eqs. (53) and (54), the weighting vectors evaluated by different "alternatives" $A_{1}, A_{2}, A_{3}, A_{4}$ can be given as:

$$
\begin{gathered}
\boldsymbol{w}^{(1)}=(0.136,0.112,0.320,0.112,0.320)^{T}, \boldsymbol{w}^{(2)}=(0.236,0.181,0.248,0.209,0.126)^{T}, \\
\boldsymbol{w}^{(3)}=(0.173,0.116,0.173,0.314,0.224)^{T}, \quad \boldsymbol{w}^{(4)}=(0.274,0.270,0.187,0.082,0.187)^{T} .
\end{gathered}
$$

So we have:

$$
\begin{gathered}
\boldsymbol{W}=\left[\boldsymbol{w}^{(1)}, \boldsymbol{w}^{(2)}, \boldsymbol{w}^{(3)}, \boldsymbol{w}^{(4)}\right]=\left[\begin{array}{llll}
0.136 & 0.236 & 0.173 & 0.274 \\
0.112 & 0.181 & 0.116 & 0.270 \\
0.320 & 0.248 & 0.173 & 0.187 \\
0.112 & 0.209 & 0.313 & 0.082 \\
0.320 & 0.126 & 0.223 & 0.187
\end{array}\right], \\
\boldsymbol{S}=\boldsymbol{W} \boldsymbol{W}^{T}=\left[\begin{array}{lllll}
0.179 & 0.152 & 0.183 & 0.141 & 0.163 \\
0.152 & 0.132 & 0.151 & 0.109 & 0.135 \\
0.183 & 0.151 & 0.229 & 0.157 & 0.207 \\
0.141 & 0.109 & 0.157 & 0.161 & 0.148 \\
0.163 & 0.135 & 0.207 & 0.148 & 0.203
\end{array}\right]
\end{gathered}
$$

The maximum eigenvalue of $S$ is 0.811 , and its corresponding vector is:

$$
\boldsymbol{w}=(0.453,0.375,0.518,0.396,0.478)^{T} .
$$

The final weighting vector for all belief structures is the normalized form of $w$, calculated as:

$$
\boldsymbol{w}^{\prime}=(0.204,0.169,0.233,0.178,0.215)^{T} \text {. }
$$




\section{Numerical examples}

We have proposed an alternative to evaluate dynamic reliability of evidence bodies, together with two evidence combination rules in the frame of intuitionistic fuzzy MCDM. The proposed reliability evaluation approach is based on self-assessment. When no a priori knowledge is available, we can evaluate evidence reliability without defining distance/similarity measure between BPAs. It can be well adapted for the fusion of highly conflicting sources of information for decision-making support. Given reliability factors /importance weights of evidence bodies, we can either combine them using intuitionistic fuzzy aggregation operators, or combine the discounted BPAs based on the combination operator of intuitionistic fuzzy values. Based on these two rules, evidence combination can be transformed to mathematical operations on IFVs. In the frame of intuitionistic fuzzy MCDM, we can choose the combination rule for them depending on the application under consideration. Two simple illustrative examples will be presented in this section to show the interest of our new reliability evaluation approach and combination rules with respect to other methods.

The context of these examples could correspond to an automatic target identification system using some independent sensors where the signals arising from these sensors are supposed to have been processed into BPAs by some given methods. The construction of BPAs is application dependent and is out of the scope of this paper. Here, we assume no prior knowledge about reliability, nor importance about the sources of evidence.

Firstly, we consider the example in [37] to show how the proposed method works for the decision making from Bayesian BPAs.

Example 5.1. Consider three simple Bayesian BPAs over the frame $\Omega=\left\{A_{1}, A_{2}, A_{3}\right\}$ as in Table 1. It is assumed that sources of evidence No.3 can provide possibly two similar BPAs denoted $m_{3 a}$ and $m_{3 b}$.

Table 1.

Three BPAs to be combined

\begin{tabular}{ccccc}
\hline & $m_{1}$ & $m_{2}$ & $m_{3 a}$ & $m_{3 b}$ \\
\hline$A_{1}$ & 0 & 0.6 & 0.75 & 0.7 \\
$A_{2}$ & 0.9 & 0.25 & 0.15 & 0.2 \\
$A_{3}$ & 0.1 & 0.15 & 0.1 & 0.1 \\
\hline
\end{tabular}

For Bayesian BPAs, the intuitionistic fuzzy MCDM model reduces to a fuzzy MCDM model. In this example, two decision matrices correspond to all BPAs can be obtained as:

$$
\begin{aligned}
& \boldsymbol{F}_{1}=\begin{array}{ccc}
m_{1} & m_{2} & m_{3 a} \\
A_{1} \\
A_{2} \\
A_{3}
\end{array}\left(\begin{array}{ccc}
<0,1> & <0.6,0.4> & <0.75,0.25> \\
<0.9,0.1> & <0.25,0.75> & <0.15,0.85> \\
<0.1,0.9> & <015,0.85> & <0.1,0.9>
\end{array}\right),
\end{aligned}
$$

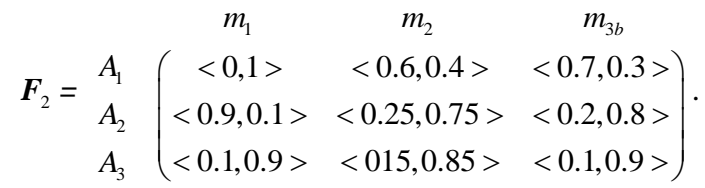

Considering BPAs $m_{1}, m_{2}$ and $m_{3 a}$, we can get their dynamic reliability factors using our proposed evaluation approach. The weighting vector is $\boldsymbol{w}_{a}=(0.294,0.411,0.295)^{T}$. If $m_{1}, m_{2}$ and $m_{3 b}$ are combined, based on $\boldsymbol{F}_{2}$ the dynamic reliability can be evaluated as $\boldsymbol{w}_{b}=(0.294,0.411,0.295)^{T}$. We can notice that the two dynamic reliability vectors are identical. That is because the probabilistic ranking result stays unchanged when sustaining $m_{3 b}$ for $m_{3 a}$.

After the determination of the discounting factors of each source, both the combination rule based on 
intuitionistic fuzzy aggregation operators and discounting combination rule based on intuitionistic fuzzy combination operator will be applied and analyzed. In this example, evidence bodies are discounted by discounting factors obtained from our proposed methods. To make our methods more transparent and comparable, the method proposed in [37] is also taken to make comparison.

The results obtained for this example are shown in Table 2. In the first row of Table 2, $m_{1}^{i}$ corresponds to the (sequential) fusion of sources $m_{1}, m_{2}, \cdots, m_{i}$. The first column of Table 2 describes the method used for combining the sources of evidence. In the second method, we discount evidence bodies by the dynamic reliability factors, and combine them using Dempster's rule. The fifth method is implemented in the frame of MCDM model, where the discounted evidence sources are combined based on the combination operation on IFVs. For clarity, combination results obtained from methods based on intuitionistic fuzzy MCDM model are directly expressed by intuitionistic fuzzy values.

Dempster's rule provides the unreasonable result that $A_{1}$ is impossible to happen, which is illogical since there are two sources among three that consider $A_{1}$ as being most possibly true. Once the discounting approach by our proposed dynamic reliability is applied before the fusion of Dempster's rule, we get the largest mass of belief to $A_{1}$ as expected. Although results of IFWA and IFWG suggest that $A_{2}$ takes the most mass of belief, they are specific for decision-making without the need of probabilistic transformation, e.g., pignistic transformation. Nevertheless, combination based on IFWG is stuck with the problem of "veto-by-one-vote", which also occurs on Dempster's rule.

We can see that $m_{2}$ and $m_{3 a}$ or $m_{3 b}$ commit most belief on $A_{1}$, and $m_{3 a}$ is very close to $m_{3 b}$, but $m_{1}$, which distributes the largest belief to $A_{2}$, highly conflicts with $m_{2}$ and $m_{3 a} / m_{3 b}$. Thus, in Liu's method, $m_{1}$ is not considered so reliable or important as the other ones according to the principle of majority. However, in our proposed self-assessment-based method, the dynamic reliability of $m_{2}$ is highest, while the reliability factor of $m_{1}$ is similar to that of $m_{3 a} / m_{3 b}$. For decision-making purpose, the fusion results presented in Table 2 and Table 3 show that all methods are sensitive to the change on the third evidence body. We can also note that the discounted \& Dempster's rue and combination operation on IFVs can work as well as Liu's method thanks to the dynamic reliability evaluation based on self-assessment.

Table 2.

Combination results of $m_{1}, m_{2}$ and $m_{3 a}$

\begin{tabular}{lllll}
\hline & $m_{1}^{3 a}\left(A_{1}\right)$ & $m_{1}^{3 a}\left(A_{2}\right)$ & $m_{1}^{3 a}\left(A_{3}\right)$ & $m_{1}^{3 a}(\Omega)$ \\
\hline Classical Dempster's rule & 0 & 0.9574 & 0.0426 & - \\
Discounted \& Dempster's rule & 0.3019 & 0.2577 & 0.0716 & 0.3599 \\
Combination based on IFWA & $<0.5438,0.4562>$ & $<0.5700,0.4300\rangle$ & $<0.1209,0.8791\rangle$ & - \\
Combination based on IFWG & $<0,1>$ & $<0.3136,0.6864>$ & $<0.1181,0.8819\rangle$ & - \\
Combination operation on IFVs & $<0.3012,0.3498>$ & $<0.2500,0.4012\rangle$ & $<0.0619,0.6270>$ & - \\
Liu's method in [37] & 0.8332 & 0.1454 & 0.0214 & \\
\hline
\end{tabular}

Table 3.

Combination results of $m_{1}, m_{2}$ and $m_{3 b}$

\begin{tabular}{lllll}
\hline & $m_{1}^{3 b}\left(A_{1}\right)$ & $m_{1}^{3 b}\left(A_{2}\right)$ & $m_{1}^{3 b}\left(A_{3}\right)$ & $m_{1}^{3 b}(\Omega)$ \\
\hline Classical Dempster's rule & 0 & 0.9677 & 0.0323 & - \\
Discounted and Dempster's rule & 0.2998 & 0.2694 & 0.0714 & 0.3594 \\
Combination based on IFWA & $<0.5187,0.4813\rangle$ & $<0.5776,0.4224>$ & $<0.1209,0.8791>$ & - \\
Combination based on IFWG & $<0,1>$ & $<0.3413,0.6587\rangle$ & $<0.1181,0.8819\rangle$ & - \\
Combination operation on IFVs & $<0.2900,0.3620\rangle$ & $<0.2617,0.3896>$ & $<0.0619,0.6270\rangle$ & - \\
Liu's method in [37] & 0.7958 & 0.1829 & 0.0213 & - \\
\hline
\end{tabular}


This example illustrate that self-assessment-based reliability evaluation method can work as well as those based on the principle of majority. Moreover, less information loss will be caused by our proposed evaluation method. Due to the difference on the principle of majority and self-assessment, different combination results can be obtained. The truth may depend on the principle we prefer to apply in the given application under consideration. This example also illustrates that combination rules based on IFWA and IFWG are not suitable for high conflicting evidence bodies.

To substantiate our arguments, we use another numerical example in [37] to demonstrate the performance of the proposed approaches when combining more than two pieces of evidence.

Example 5.2. Let's consider a set of five normalized BPAs with imprecise focal elements over the frame of discernment $\Omega=\left\{A_{1}, A_{2}, A_{3}\right\}$ as given in Table 4. In this example $m_{3}$ is a Bayesian BPA, whereas all other BPAs are non-Bayesian.

Table 4.

Five BPAs to be combined

\begin{tabular}{llllll}
\hline & $m_{1}$ & $m_{2}$ & $m_{3}$ & $m_{4}$ & $m_{5}$ \\
\hline$A_{1}$ & 0.8 & 0.4 & 0 & 0.3 & 0.45 \\
$A_{2}$ & 0.1 & 0.2 & 0.95 & 0.2 & 0.1 \\
$A_{3}$ & 0 & 0.1 & 0.05 & 0.25 & 0 \\
$\left\{A_{1}, A_{2}\right\}$ & 0 & 0.3 & 0 & 0.2 & 0 \\
$\left\{A_{2}, A_{3}\right\}$ & 0 & 0 & 0 & 0 & 0.15 \\
$\Omega$ & 0.1 & 0 & 0 & 0.05 & 0.3 \\
\hline
\end{tabular}

When we use our proposed methods to determine the weights of evidence sources, decision matrices are needed. For convenience, we first give the intuitionistic fuzzy MCDM model $\boldsymbol{F}$ as shown in Eq. (56) based on all BPAs. We should extract different columns to yield decision matrix according to evidence bodies to be combined.

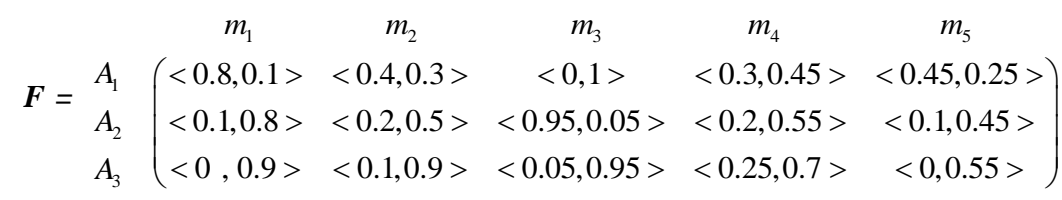

Table 5 shows the combination of all the sources. We can see that when the first two evidence sources are combined, all methods can derive the result the hypothesis $A_{1}$ is very unlikely to happen. But when $m_{3}$ is considered, $m\left(A_{1}\right)$ decreases while $m\left(A_{2}\right)$ increases for all methods. Moreover, Dempster's rule, IFWA-based combination and IFWG-based combination present opposite conclusion. That is because BPAs $m_{1}, m_{2}, m_{4}$ and $m_{5}$ assign most of their belief to $A_{1}$, but $m_{3}$ oppositely commits its largest mass of belief to $A_{2}$. By our proposed reliability evaluation method, we can get $\boldsymbol{w}=[0.295,0.411,0294]^{T}$. The reliability factor of $m_{3}$ is close to that of $m_{1}$. So the combination rule based on IFWA and IFWG cannot reduce the influence of $m_{3}$, whereas the discounting operation is competent enough to reduce its affect. With the participation of other evidence sources supporting $A_{1}$, the probability assigned to $A_{2}$ decreases, which is better to decision making. It is worth noticing that the classical Dempster's rule and the combination based on IFWG are easy to fall into "veto-by-one-vote".

In this example, we can note that the dynamic reliability factor estimated based on self-assessment is sensitive to the change of evidence sources. This reliability factor can be applied to discount evidence to reduce the influence of unreliable evidence bodies. We can also find that combining discounted evidence sources based on combination operation on IFVs is much more convenient to get the probability distribution on the discernment frame. Moreover, results expressed by IFVs can be probabilistically compared to get soft decision. In some applications, certain risk always hides in a crisp decision, while soft decision may be more helpful. 
The interest of the new reliability estimation method lies in the principle of self-assessment, where each criterion is regarded as an estimation agent. The debatable distance/similarity measure between evidence bodies is unnecessary in the proposed evaluation method. The combination rules based on IFWA and IFWG are not as applicable as the discounting operation on unreliable evidences. When combining discounted evidence sources, both classical Dempster's rule and combination operation on IFVs can be used. However, we find that the latter is more efficient without transforming belief functions to Bayesian probabilities.

Table 5.

Combination results of different evidence bodies

\begin{tabular}{|c|c|c|c|c|}
\hline & $m_{1}^{2}$ & $m_{1}^{3}$ & $m_{1}^{4}$ & $m_{1}^{5}$ \\
\hline Classical Dempster's rule & $\begin{array}{l}m\left(A_{1}\right)=0.8451 \\
m\left(A_{2}\right)=0.0986 \\
m\left(A_{3}\right)=0.0140 \\
m\left(A_{1}, A_{2}\right)=0.0423\end{array}$ & $\begin{array}{l}m\left(A_{1}\right)=0 \\
m\left(A_{2}\right)=0.9948 \\
m\left(A_{3}\right)=0.0052\end{array}$ & $\begin{array}{l}m\left(A_{1}\right)=0 \\
m\left(A_{2}\right)=0.9965 \\
m\left(A_{3}\right)=0.0035\end{array}$ & $\begin{array}{l}m\left(A_{1}\right)=0 \\
m\left(A_{2}\right)=0.9971 \\
m\left(A_{3}\right)=0.0029\end{array}$ \\
\hline Discounted and Dempster's rule & $\begin{array}{l}m\left(A_{1}\right)=0.4451 \\
m\left(A_{2}\right)=0.1136 \\
m\left(A_{3}\right)=0.0420 \\
m\left(A_{1}, A_{2}\right)=0.1261 \\
m\left(A_{2}, A_{3}\right)=0 \\
m(\Omega)=0.2732\end{array}$ & $\begin{array}{l}m\left(A_{1}\right)=0.2694 \\
m\left(A_{2}\right)=0.2671 \\
m\left(A_{3}\right)=0.0328 \\
m\left(A_{1}, A_{2}\right)=0.0746 \\
m\left(A_{2}, A_{3}\right)=0 \\
m(\Omega)=0.3561\end{array}$ & $\begin{array}{l}m\left(A_{1}\right)=0.2428 \\
m\left(A_{2}\right)=0.2350 \\
m\left(A_{3}\right)=0.0585 \\
m\left(A_{1}, A_{2}\right)=0.0793 \\
m\left(A_{2}, A_{3}\right)=0 \\
m(\Omega)=0.3843\end{array}$ & $\begin{array}{l}m\left(A_{1}\right)=0.2628 \\
m\left(A_{2}\right)=0.1935 \\
m\left(A_{3}\right)=0.0460 \\
m\left(A_{1}, A_{2}\right)=0.0586 \\
m\left(A_{2}, A_{3}\right)=0.0177 \\
m(\Omega)=0.4214\end{array}$ \\
\hline Combination based on IFWA & $\begin{array}{l}m\left(A_{1}\right)= \\
<0.6108,0.1946> \\
m\left(A_{2}\right)= \\
<0.1620,0.6017> \\
m\left(A_{3}\right)= \\
<0.0619,0.9000>\end{array}$ & $\begin{array}{l}m\left(A_{1}\right)= \\
<0.4953,0.3095> \\
m\left(A_{2}\right)= \\
<0.6339,0.2915> \\
m\left(A_{3}\right)= \\
<0.0567,0.9144>\end{array}$ & $\begin{array}{l}m\left(A_{1}\right)= \\
<0.4411,0.3503> \\
m\left(A_{2}\right)= \\
<0.5650,0.3342> \\
m\left(A_{3}\right)= \\
<0.1130,0.8518>\end{array}$ & $\begin{array}{l}m\left(A_{1}\right)= \\
<0.4509,0.3184> \\
m\left(A_{2}\right)= \\
<0.4782,0.3633> \\
m\left(A_{3}\right)= \\
<0.0869,0.7685>\end{array}$ \\
\hline Combination based on IFWG & $\begin{array}{l}m\left(A_{1}\right)= \\
<0.5256,0.2272> \\
m\left(A_{2}\right)= \\
<0.1522,0.6515> \\
m\left(A_{3}\right)= \\
<0,0.9000>\end{array}$ & $\begin{array}{l}m\left(A_{1}\right)=\langle 0,1> \\
m\left(A_{2}\right)= \\
<0.2580,0.5388> \\
m\left(A_{3}\right)= \\
<0,0.9185>\end{array}$ & $\begin{array}{l}m\left(A_{1}\right)=\langle 0,1> \\
m\left(A_{2}\right)= \\
<0.2471,0.5350> \\
m\left(A_{3}\right)= \\
<0,0.8854>\end{array}$ & $\begin{array}{l}m\left(A_{1}\right)=\langle 0,1> \\
m\left(A_{2}\right)= \\
<0.1973,0.5223> \\
m\left(A_{3}\right)= \\
<0,0.8416>\end{array}$ \\
\hline Combination operation on IFVs & $\begin{array}{l}m\left(A_{1}\right)= \\
<0.4440,0.1578> \\
m\left(A_{2}\right)= \\
<0.1113,0.4975> \\
m\left(A_{3}\right)= \\
<0.0400,0.7002>\end{array}$ & $\begin{array}{l}m\left(A_{1}\right)= \\
<0.2656,0.3097> \\
m\left(A_{2}\right)= \\
<0.2630,0.3127> \\
m\left(A_{3}\right)= \\
<0.0289,0.6572>\end{array}$ & $\begin{array}{l}m\left(A_{1}\right)= \\
<0.2354,0.3151> \\
m\left(A_{2}\right)= \\
<0.2278,0.3228> \\
m\left(A_{3}\right)= \\
<0.0531,0.5985>\end{array}$ & $\begin{array}{l}m\left(A_{1}\right)= \\
<0.2580,0.2707> \\
m\left(A_{2}\right)= \\
<0.1850,0.3308> \\
m\left(A_{3}\right)= \\
<0.0420,0.5576>\end{array}$ \\
\hline Liu's method in [37] & $\begin{array}{l}m\left(A_{1}\right)=0.7503 \\
m\left(A_{2}\right)=0.1196 \\
m\left(A_{3}\right)=0.0319 \\
m\left(A_{1}, A_{2}\right)=0.0957 \\
m(\Omega)=0.0025\end{array}$ & $\begin{array}{l}m\left(A_{1}\right)=0.7157 \\
m\left(A_{2}\right)=0.1598 \\
m\left(A_{3}\right)=0.0308 \\
m\left(A_{1}, A_{2}\right)=0.0913 \\
m(\Omega)=0.0024\end{array}$ & $\begin{array}{l}m\left(A_{1}\right)=0.7670 \\
m\left(A_{2}\right)=0.11655 \\
m\left(A_{3}\right)=0.0194 \\
m\left(A_{1}, A_{2}\right)=0.0477 \\
m(\Omega)=0.0004\end{array}$ & $\begin{array}{l}m\left(A_{1}\right)=0.8254 \\
m\left(A_{2}\right)=0.1424 \\
m\left(A_{3}\right)=0.0120 \\
m\left(A_{1}, A_{2}\right)=0.0198 \\
m\left(A_{2}, A_{3}\right)=0.0002 \\
m(\Omega)=0.0002\end{array}$ \\
\hline
\end{tabular}

The performance of all methods has been illustrated by above numerical examples. In the application of information fusion based on evidence theory, the dynamic reliability of unreliable evidence sources can be evaluated by our proposed method based on self-assessment. Then the BPAs corresponding to evidence sources can be discounted by Shafer's discounting rule. When combining discounted BPAs, Dempster's combination rule and the combination operation between IFVs can both obtain rational results. However, it is more sensible to choose the latter for the convenience it brings for decision making. 


\section{Conclusion and further study}

In this paper we mainly investigated the issues of combination of unreliable evidence sources in the frame of multiple criteria decision making model under intuitionistic fuzzy environment. By transforming the problem of evidence combination to MCDM model in intuitionistic fuzzy environment, we can combine evidence sources based on intuitionistic fuzzy aggregation operators IFWA and IFWG when the reliability factors are known. As for intuitionistic fuzzy weights, evidence bodies can be combined by G-IFWA and G-IFWG operators. Considering the limitation of intuitionistic fuzzy aggregation operators, we also generalize discounting operation on evidence sources to address the problem of uncertain reliability factors. We prove that the generalized discounting operation reduces to Shafer's discounting operation when reliability factors are real numbers. In most applications, a priori knowledge is unknown, so we need to evaluate the dynamic reliability of evidence sources. Unlike many existing reliability evaluation method, our proposed method is based on the principle of self-assessment and the probabilistic comparison between intuitionistic fuzzy values. Numerical examples demonstrate that the proposed method is capable of reducing the influence of unreliable evidence sources. Moreover, a combination operation on intuitionistic fuzzy values is defined to combine evidence bodies in MCDM model. It has been illustrated that combining discounted evidence sources based combination operation on IFVs is competent to deal with conflicting evidence bodies. In addition, we can make soft decision based on intuitionistic fuzzy valued fusion results, which is more cautious (less risky) in many applications.

Finally, we must point out the combination rules based on intuitionistic fuzzy aggregation operators are not efficient enough for high conflicting evidence sources. When we combine evidence bodies based on IFAG operator, it is easy to fall into "veto-by-one-vote". Generally, the combination method based on our proposed combination operation on IFVs is more competent. Another issue worth to be mentioned is the computational complexity of our proposed combination operation. Since our defined combination operation on IFVs are all based on arithmetic operations, which are much simpler than the nonlinear optimal operation. Therefore, computational complexity is not a problem for the proposed operation.

\section{Acknowledgments}

This work is supported by National Science Foundation of China under grants 60975026 and 61273275

\section{References}

[1] A.P. Dempster, Upper and lower probabilities induced by a multi-valued mapping, Ann. Math. Statist. 38 (1967) $325-339$

[2] G. Shafer, A Mathematical Theory of Evidence, Princeton Univ. Press, 1976

[3] G. Shafer, Perspectives on the theory and practice of belief functions, Int. J. Approx. Reason. 4 (5-6) (1990) $323-362$.

[4] Z. Liu, Q. Pan, J. Dezert, Evidential classifier for imprecise data based on belief functions, Knowl.-Based Syst. 52 (2013) 246-257.

[5] P. Xu, Y. Deng, X. Su, Sankaran Mahadevan: a new method to determine basic probability assignment from training data, Knowl.-Based Syst. 46 (2013) 69-80

[6] T. Denoeux, M.-H. Masson, EVCLUS: evidential clustering of proximity data, IEEE Trans. Syst. Man Cybernet. Part B 34 (1) (2004) 95-109.

[7] M.-H. Masson, T. Denoeux, Clustering interval-valued data using belief functions, Pattern Recogn. Lett. 25 (2) (2004) 163-171

[8] M.-H. Masson, T. Denoeux, Ensemble clustering in the belief functions framework, Int. J. Approx. Reason. 52 (1) (2011) 92-109.

[9] M.-H. Masson, T. Denoeux, ECM: an evidential version of the fuzzy c-means algorithm, Pattern Recogn. 41 (4) (2008) 1384-1397.

[10] D. Han, Y. Deng, C. Han, Sequential weighted combination for unreliable evidence based on evidence variance, Decis. Support Syst. 56 (2013) 387-393.

[11] S. Huang, X. Su, Y. Hu, S. Mahadevan, Y. Deng, A new decision-making method by incomplete preferences based on evidence distance, Knowl.-Based Syst. 56 (2014) 264-272.

[12] Z. Liu, J. Dezert, G. Mercier, Q. Pan, Dynamic evidential reasoning for change detection in remote sensing images, IEEE Trans. Geosci. Remote Sens. 50 (5) (2012) 1955-1967.

[13] Z. Liu, J. Dezert, G. Mercier, Q. Pan, Belief c-means: an extension of fuzzy c-means algorithm in belief functions framework, Pattern Recogn. Lett. 33 (3) (2012) 291-300. 
[14] F. Klawonn, E. Schwecke, On the axiomatic justification of Dempster's rule of combination, Inter. J. Intell. Syst. 7 (5) (1992) 469-478.

[15] D. Dubois, H. Prade, On the unicity of Dempster rule of combination, Inter. J. Intell. Syst. 1 (1986) 133-142.

[16] F. Pichon, T. Denoeux, The unnormalized Dempster's rule of combination: a new justification from the Least Commitment Principle and some extensions, J. Autom. Reason. 45 (1) (2010) 61-87.

[17] L.A. Zadeh, A simple view of the Dempster-Shafer theory of evidence and its implication for the rule of combination, AI Mag. 2 (1986) 85-90.

[18] R. Haenni, Shedding new light on Zadeh's criticism of Dempster's rule of combination, in: Proceedings of the Eighth International Conference on Information Fusion, Philadelphia, USA, IEEE, Piscataway, NJ, 2005, pp. 879-884.

[19] F. Smarandache, J. Dezert, Applications and Advances of DSmT for Information Fusion, vol. 3, American Research Press, Rehoboth, 2009, pp. 4-32.

[20] E. Lefevre, O. Colot, P. Vannoorenberghe, Belief functions combination and conflict management, Information Fusion 3 (2) (2002) 149-162.

[21] P. Smets, Data fusion in the transferable belief model, in: Proceedings of the 3rd International Conference on Information Fusion, Paris, France, 2000, pp.PS21-PS33.

[22] M.C. Florea, A.-L. Jousselme, E. Bosse, Robust combination rules for evidence theory, Inform. Fusion 10 (2) (2009) 183-197.

[23] R. R. Yager, On the Dempster-Shafer framework and new combination rules, Inform. Sci. 41 (2) (1987) 93-137.

[24] C. K. Murphy, Combining belief functions when evidence conflicts, Decis. Support Syst. 29 (1) (2000) 1-9.

[25] Y. Deng, W.K. Shi, Z.F. Zhu, Q. Liu, Combining belief functions based on distance of evidence, Decis. Support Syst. 38 (3) (2004) 489-493.

[26] J. Klein, O. Colot, Automatic discounting rate computation using a dissent criterion, in: Proceedings of the Workshop on the Theory of Belief Functions, Brest, France, 2010, pp. 1-6.

[27] Y. Yang, D. Han, C. Han, Discounted combination of unreliable evidence using degree of disagreement, Int. J. Approx. Reason. 54 (2013) 1197-1216.

[28] L. A. Zadeh, Fuzzy sets, Inform. Control 8 (1965) 338-353.

[29] K. T. Atanassov, Intuitionistic fuzzy sets, Fuzzy Sets Syst. 20 (1) (1986) 87-96

[30] K. T. Atanassov, On intuitionistic fuzzy sets theory, Berlin: Springer-Verlag; 2012.

[31] H. Bustince, P. Burillo, Vague sets are intuitionistic fuzzy sets, Fuzzy Sets Syst. 79 (3) (1996) 403-405.

[32] K. T. Atanassov, G. Gargov, Interval-valued intuitionistic fuzzy sets, Fuzzy Sets Syst. 31 (3) (1989) 343-349.

[33] Z. Elouedi, K. Mellouli, P. Smets, Assessing Sensor Reliability for Multisensor Data Fusion Within the Transferable Belief Model, IEEE Trans. Syst. Man Cybernet. Part B 34(4) (2004) 782-787.

[34] H. Guo, W. Shi, Y. Deng, Evaluating Sensor Reliability in Classification Problems Based on Evidence Theory, IEEE Trans. Syst. Man Cybernet. Part B 36(5) (2006) 970-981.

[35] J. Klein, O. Colot, Automatic discounting rate computation using a dissent criterion, in: Proceedings of the Workshop on the Theory of Belief Functions, Brest, France, 2010, pp. 1-6.

[36] A.-L. Jousselme, D. Grenier, E. Bosse, A new distance between two bodies of evidence, Inform. Fusion 2 (2) (2001) 91-101.

[37] Z. Liu, J. Dezert, Q. Pan, G. Mercier, Combination of sources of evidence with different discounting factors based on a new dissimilarity measure, Decis. Support Syst. 52 (2011) 133-141.

[38] J. Schubert, Conflict management in Dempster-Shafer theory using the degree of falsity, International Journal of Approximate Reasoning 52 (3) (2011) 449-460.

[39] G. Zhang, J. Ma, J. Lu. Emergency management evaluation by a fuzzy multi-criteria group decision support system, Stoch Environ Res Risk Assess 23 (4) (2009) 517-527.

[40] J. Lu, J. Ma, G. Zhang, Y. Zhu, X. Zeng, L. Koehl, Theme-based comprehensive evaluation in new product development using fuzzy hierarchical criteria group decision-making method, IEEE Trans. Indus. Elec. 58(6) (2011) 2236-2246.

[41] G.-W. Wei, GRA method for multiple attribute decision making with incomplete weight information in intuitionistic fuzzy setting, Knowledge-Based Systems 23 (2010) 243-247.

[42] G.-W. Wei, Maximizing deviation method for multiple attribute decision making in intuitionistic fuzzy setting, Knowledge-Based Systems 21 (2008) 833-836.

[43] G.-W. Wei, Gray relational analysis method for intuitionistic fuzzy multiple attribute decision making, Expert Systems with Applications 38 (2011) 11671-11677.

[44] G. Beliakov, H. Bustince, D.P. Goswami, U.K. Mukherjee, N.R. Pal, On averaging operators for Atanassov's intuitionistic fuzzy sets, Inform. Sci. 181(2011) 1116-1124.

[45] Z. Xu, Intuitionistic fuzzy aggregation operators, IEEE Trans. Fuzzy Syst. 15 (2007) 1179-1187.

[46] Z. Xu, R.R. Yager, Some geometric aggregation operators based on intuitionistic fuzzy sets, Inter. J. Gen. Syst. 35 (2006) 417-433

[47] B. Li, W. He, Intuitionistic fuzzy PRI-AND and PRI-OR aggregation operators, Inform. Fusion 14 (2013) 450-459.

[48] W. L. Gau, D.J. Buehrer, Vague sets, IEEE Trans. Syst. Man Cybernet. 23 (1993) 610-614.

[49] S. M. Chen, J.M. Tan, Handling multicriteria fuzzy decision-making problems based on vague set theory, Fuzzy Sets Syst. 67 (1994) $163-172$

[50] D. H. Hong, C.H. Choi, Multicriteria fuzzy decision-making problems based on vague set theory, Fuzzy Sets Syst. 114 (2000) 103-113.

[51] D.-F. Li, The GOWA operator based approach to multiattribute decision making using intuitionistic fuzzy sets, Math. Comput. Model. 53 (2011) 1182-1196.

[52] Y.-M. Wang, T. M. S. Elhag, On the normalization of interval and fuzzy weights, Fuzzy Sets Syst. 157 (2006) 2456-2471.

[53] L. Dymova, P. Sevastjanov, An interpretation of intuitionistic fuzzy sets in terms of evidence theory: Decision making aspect, Knowl.-Based Syst. 23 (2010) 772-782.

[54] J.-B. Yang, D.-L. Xu, Evidential reasoning rule for evidence combination, Artif. Intell. 205 (2013) 1-29. 
[55] F. Smarandache, J. Dezert, J.M. Tacnet, Fusion of sources of evidence with different importances and reliabilities, in: The 2010 13th IEEE Conference on Information Fusion (FUSION), 2010, pp. 1-8.

[56] Y. Song, X. Wang, L. Lei, A. Xue, Combination of interval-valued belief structures based on intuitionistic fuzzy set, Knowl.-Based Syst. 67 (2014) 61-70.

[57] L.-H. Chen, C.-C. Hung, C.-C. Tu, Considering the decision maker's attitudinal character to solve multi-criteria decision-making problems in an intuitionistic fuzzy environment, Knowl.-Based Syst. 36 (2012) 129-138.

[58] Z. Xu, An Overview of Methods for Determining OWA Weights, Inter. J. Intell.t Syst. 20 (2005) 843-865.

[59] Z. Xu, Q. L. Da, The Uncertain OWA Operator, Inter. J. Intell.t Syst. 17 (2002) 569-575.

[60] Y. Nakahara, User oriented ranking criteria and its application to fuzzy mathematical programming problems, Fuzzy Sets Syst. 94 (1998) 275-276

[61] Y. Nakahara, M. Sasaki, M. Gen, On the linear programming problems with interval coefficients, Comput. Ind. Eng. 23 (1992) 301-304.

[62] G. Facchinetti, R. Ricci, S. Muzzioli, Note on ranking fuzzy triangular numbers, Inter. J. Intell.t Syst. 13 (1998) 613-622.

[63] A. Frikha, On the use of a multi-criteria approach for reliability estimation in belief function theory, Inform. Fusion 18 (2014) 20-32. 